\title{
Two-loop fermion self-energy in reduced quantum electrodynamics and application to the ultra-relativistic limit of graphene
}

\author{
A. V. Kotikov ${ }^{1}$ and S. Teber ${ }^{2,3}$ \\ ${ }^{1}$ Bogoliubov Laboratory of Theoretical Physics, Joint Institute for Nuclear Research, 141980 Dubna, Russia. \\ ${ }^{2}$ Sorbonne Universités, UPMC Univ Paris 06, UMR 7589, LPTHE, F-75005, Paris, France. \\ ${ }^{3}$ CNRS, UMR 7589, LPTHE, F-75005, Paris, France.
}

(Dated: February 18, 2018)

\begin{abstract}
We compute the two-loop fermion self-energy in massless reduced quantum electrodynamics for an arbitrary gauge using the method of integration by parts. Focusing on the limit where the photon field is four-dimensional, our formula involves only recursively one-loop integrals and can therefore be evaluated exactly. From this formula, we deduce the anomalous scaling dimension of the fermion field as well as the renormalized fermion propagator up to two loops. The results are then applied to the ultra-relativistic limit of graphene and compared with similar results obtained for four-dimensional and three-dimensional quantum electrodynamics.
\end{abstract}

\section{INTRODUCTION}

In condensed matter physics, an emergent relativity at low energies appears for systems with two stable Fermi points, see, e.g., the textbook Ref. 11. This is the case of undoped graphene, a one-atom thick layer of graphite, see, e.g., Ref. [2] for a review, where the quasiparticle spectrum is Dirac-like and massless at low-energies 314 . In Ref. [5] a renormalization group approach indeed revealed the existence of an infrared Lorentz invariant fixed point for graphene. Approaching this fixed point, the Fermi velocity, $v_{F}$, flows to the velocity of light, $c \approx 300 v_{F}$, while the fine structure constant of graphene, $\alpha_{g} \approx e^{2} / 4 \pi \varepsilon \hbar v_{F}$, which is of order one, flows to the usual fine structure constant, $\alpha \approx 1 / 137$. Moreover, while electrons in graphene are confined to a three-dimensional spacetime, $d_{e}=2+1$, interactions between them are mediated by four-dimensional photons, $d_{\gamma}=3+1$. The Lorentz invariant fixed point may therefore be effectively described by a massless relativistic quantum field theory (QFT) model whereby $2+1$-dimensional electrons interact via a long-range fully retarded potential. Such a model belongs to the class of reduced quantum electrodynamics (RQED) 6, or $\mathrm{RQED}_{d_{\gamma}, d_{e}}$, and corresponds to $\mathrm{RQED}_{4,3}$ in the case of graphene. Following Ref. [7, the latter is also sometimes referred to as pseudo-QED, see, e.g., Ref. [8]. In the general case, $\mathrm{RQED}_{d_{\gamma}, d_{e}}$ is a relativistic QFT describing the interaction of an abelian $U(1)$ gauge field living in $d_{\gamma}$ space-time dimensions with a fermion field living in a space-time of $d_{e}$ dimensions. The case where $d_{e}=d_{\gamma}$ corresponds to usual QEDs, see the textbooks Refs. 9-11. The reduced case corresponds to $d_{e}<d_{\gamma}$ where the fermion field is localized on a $\left(d_{e}-1\right)$ brane. Motivated by potential applications to condensed matter physics as well as interest in branes we focus on the computation of radiative corrections in a general

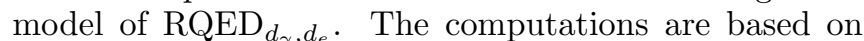
sophisticated methods devoted to the exact evaluation of multi-loop Feynman diagrams in relativistic QFTs; these methods include, e.g., the Gegenbauer polynomial technique ${ }^{12 \mid 13}$, integration by parts ${ }^{14 \mid 15}$ (IBP), and the method of uniqueness 1416

In Refs. 2021 multi-loop corrections in a general theory of $\mathrm{RQED}_{d_{\gamma}, d_{e}}$ were computed with a special emphasis on electromagnetic current correlations and the link between $\mathrm{RQED}_{4,3}$ and the ultrarelativistic limit of graphene was made. In particular, the two-loop interaction correction coefficient to the polarization operator of graphene in the ultrarelativistic limit was derived and found to be small in qualitative agreement with theoretical $\sqrt{22}$ as well as experimenta $\sqrt{23}$ results in the non-relativistic regime. These results were used in Ref. 24 to show that the optical conductivity of graphene in the ultrarelativistic limit has a relative deviation which is within experimental uncertainty ${ }^{23}$ and of the order of one percent with respect to the non-interacting value. This striking qualitative agreement with the non relativistic limit together with the efficient tools available to tackle the relativistic limit suggest the crucial importance of fully exploring the properties of the Lorentz invariant fixed point.

We pursue this task in the present paper by computing multi-loop corrections to the fermion propagator and the corresponding anomalous scaling dimension of the fermion field in $\mathrm{RQED}_{4, d_{e}}$. A peculiarity of graphene is that the fermion field gets renormalized but not the electric charge, see Ref. [5]. A similar feature was found for $\mathrm{RQED}_{4,3}$ in Ref. [20] where the anomalous dimension of the fermion field was computed at one-loop. In the following we extend these results to two loops. From the field theory point of view, the main technical difficulty involves the computation of some peculiar two-loop massless propagator diagrams with two non-integer indices on non-adjacent lines (see Eq. (39) below). Presently, no explicit analytical expression for such diagrams is known. It turns out that, as will be shown below, repeated use of some well chosen IBP identities allows us to express each complicated, and eventually divergent, diagram as a sum of primitive (or recursively one-loop) diagrams plus a complicated but convergent diagram which is multiplied by a factor $\epsilon_{\gamma}=2-d_{\gamma} / 2$. In the limit, $d_{\gamma} \rightarrow 4$, the complicated part does not contribute which solves the problem in the case of $\mathrm{RQED}_{4, d_{e}}$.

The paper is organized as follows. In Sec. II], we review 
the basics of massless RQED at one-loop. In Sec. III] we compute the two-loop fermion self-energy in $\mathrm{RQED}_{d_{\gamma}, d_{e}}$ using IBP identities and show that, for $d_{\gamma} \rightarrow 4$, it can be expressed only as a function of recursively one-loop diagrams. In Sec. IV] we compute the anomalous scaling dimension of the fermion field and the renormalized fermion propagator at two-loop, in an arbitrary gauge in the general case of $\mathrm{RQED}_{4, d_{e}}$. The special case of $\mathrm{RQED}_{4,3}$ is then explicitly considered as well as, for completeness, the cases of $\mathrm{QED}_{4}$ and $\mathrm{QED}_{3}$. In Sec. $\mathrm{V}$ we summarize our results and conclude. Finally, App. A contains the expansion of some master integrals entering the two-loop self-energy and App. B presents general formulas, valid beyond IBP relations, for diagrams with two non-integer indices. In the following, we work in units where $\hbar=c=1$.

\section{MASSLESS RQED}

\section{A. The model}

We consider a general model of massless $\mathrm{RQED}_{d_{\gamma}, d_{e}}$ described by $6 \mid 20$ :

$$
\begin{aligned}
S_{\mathrm{RQED}}= & \int \mathrm{d}^{d_{\gamma}} x \mathcal{L}_{\mathrm{RQED}}, \\
\mathcal{L}_{\mathrm{RQED}}= & \bar{\psi}(x) i \gamma^{\mu_{e}} D_{\mu_{e}} \psi(x) \delta^{\left(d_{\gamma}-d_{e}\right)}(x)- \\
& -\frac{1}{4} F_{\mu_{\gamma} \nu_{\gamma}} F^{\mu_{\gamma} \nu_{\gamma}}-\frac{1}{2 a}\left(\partial_{\mu_{\gamma}} A^{\mu_{\gamma}}\right)^{2},
\end{aligned}
$$

where $D_{\mu}=\partial_{\mu}+i e A_{\mu}$ is the covariant derivative, $F_{\mu \nu}=\partial_{\mu} A_{\nu}-\partial_{\nu} A_{\mu}$ is the field stress tensor of the gauge potential $A^{\mu}$ and $a$ is a gauge fixing parameter. In Eq. (1), on the one-hand, the index $\mu_{e}$ runs over the $d_{e}$-dimensional space-time in which fermions are localized: $\mu_{e}=0,1, \ldots, d_{e}-1$, where zero index corresponds to time. On the other hand, the index $\mu_{\gamma}$ runs over the $d_{\gamma}$-dimensional space-time of the gauge field: $\mu_{\gamma}=0,1, \ldots, d_{e}-1, d_{e}, \ldots, d_{\gamma}-1$, where we assume that $d_{\gamma} \geq d_{e}$. The minimal coupling of the gauge field to the fermion current, $j_{\mu} A^{\mu}$, is restricted to the reduced matter space from which we deduce the expression of the fermion current:

$j^{\mu}(x)= \begin{cases}\mathrm{e} \bar{\psi}(x) \gamma^{\mu} \psi(x) \delta^{\left(d_{\gamma}-d_{e}\right)}(x) & \mu=0, \cdots, d_{e}-1 \\ 0 & \mu=d_{e}, \cdots, d_{\gamma}-1 .\end{cases}$

Simple dimensional analysis then shows that two epsilon parameters are naturally associated with the two dimensions $d_{\gamma}$ and $d_{e}$. Indeed, for a general $\mathrm{RQED}_{d_{\gamma}, d_{e}}$, the dimensions of the fields and electric charge may be written as:

$$
\left[A_{\mu}\right]=1-\varepsilon_{\gamma}, \quad[\psi]=\frac{3}{2}-\varepsilon_{e}-\varepsilon_{\gamma}, \quad[e]=\varepsilon_{\gamma},
$$

where the two epsilon parameters read:

$$
\varepsilon_{\gamma}=\frac{4-d_{\gamma}}{2}, \quad \varepsilon_{e}=\frac{d_{\gamma}-d_{e}}{2},
$$

\begin{tabular}{|l||c|c|c|}
\hline & $\mathrm{QED}_{4}$ & $\mathrm{RQED}_{4,3}$ & $\mathrm{RQED}_{4,2}$ \\
\hline \hline Photon self-energy & $2(0)$ & $1(-1)$ & $0(-2)$ \\
\hline Fermion self-energy & $1(0)$ & $1(0)$ & $1(0)$ \\
\hline Fermion-photon vertex & $0(0)$ & $0(0)$ & $0(0)$ \\
\hline
\end{tabular}

TABLE I: Superficial degree of divergence (and effective degree of divergence) of the three most divergent amplitudes in $\mathrm{RQED}_{4, d_{e}}$ for $d_{e}=4\left(\mathrm{QED}_{4}\right), d_{e}=3$ and $d_{e}=2$.

and will be shown below to play a crucial role in setting up a dimensional regularization scheme for RQED. Conversely, Eq. (4) yields the relations:

$$
d_{\gamma}=4-2 \varepsilon_{\gamma}, \quad d_{e}=4-2 \varepsilon_{e}-2 \varepsilon_{\gamma} .
$$

As can be seen from Eq. (3) the dimension of the coupling constant is entirely determined by the space-time dimension of the gauge field, $d_{\gamma}$, or, equivalently, $\varepsilon_{\gamma}$; for a four-dimensional gauge field $\left(d_{\gamma}=4\right)$ the coupling is dimensionless so that, at least at a classical level, the theory is scale invariant. This suggests that $\mathrm{RQED}_{4, d_{e}}$ are renormalizable QFTs. Following Ref. [20, let's recall that the superficial degree of divergence (SDD) of a general $\mathrm{RQED}_{d_{\gamma}, d_{e}}$ diagram reads: $\underline{37}$

$$
D=d_{e}+\frac{d_{\gamma}-4}{2} V-\frac{d_{\gamma}-2}{2} N_{\gamma}-\frac{d_{e}-1}{2} N_{e},
$$

where $V$ is the number of vertices, $N_{\gamma}$ the number of external gauge lines and $N_{e}$ the number of external fermion lines. From Eq. (6) we see that for $d_{\gamma}=4$ the SDD does not depend on the number of vertices whatever value $d_{e}$ takes; this confirms the renormalizability of the theory. A peculiar fact of reduced theories is that, while the fermion self-energy and fermion-photon vertex have the same degree of divergence as in usual $\mathrm{QED}_{4}$ (they effectively diverge logarithmically), the photon self-energies of $\mathrm{RQED}_{4,3}$ and $\mathrm{RQED}_{4,2}$ are finite. This is summarized in Tab. I which displays the degrees of divergence (superficial and effective) of the three most divergent amplitudes in $\mathrm{QED}_{4}, \mathrm{RQED}_{4,3}$ and $\mathrm{RQED}_{4,2}$. As a consequence, while the fermion field acquires an anomalous dimension, the coupling constant does not renormalize.

\section{B. Perturbation theory and renormalization}

The above dimensional analysis can be made quantitative by setting up a perturbative approach to RQED. As can be seen from Eq. (1) the free massless fermion propagator is the usual one and reads:

$$
S_{0}\left(p_{e}\right)=\frac{i \not p}{p^{2}+i 0^{+}},
$$

where $p=p_{0}, \ldots, p_{d_{e}-1}$ lies in the reduced matter space and $i 0^{+}$is a convergence factor that will often be omitted 
in the following in order to simplify notations. On the other hand, because we are interested in the properties of the reduced system, we may integrate over the $d_{\gamma}-d_{e}$ bulk gauge degrees of freedom. This yields an effective free gauge field propagator reading:

$$
\tilde{D}_{0}^{\mu \nu}(q)=\frac{i}{(4 \pi)^{\varepsilon_{e}}} \frac{\Gamma\left(1-\varepsilon_{e}\right)}{\left(-q^{2}\right)^{1-\varepsilon_{e}}}\left(g^{\mu \nu}-\tilde{\xi} \frac{q^{\mu} q^{\nu}}{q^{2}}\right),
$$

where, now, $q=q_{0}, \ldots, q_{d_{e}-1}$ lies in the reduced matter space and $\varepsilon_{e}$ was defined in Eq. (4). Moreover, we see that the gauge fixing parameter is affected by the integration of the bulk gauge modes: $\tilde{\xi}=\xi\left(1-\varepsilon_{e}\right)$ where $\xi=1-a$. The two commonly used relativistic gauges are the Feynman and Landau gauges which are defined as:

$$
\begin{array}{rlll}
\text { Feynman gauge : } & \xi=0 & \text { or } & a=1, \\
\text { Landau gauge : } & \xi=1 & \text { or } & a=0 .
\end{array}
$$

In the following we shall mainly work in an arbitrary gauge.

In the case of $\mathrm{RQED}_{4,3}$ for which $\varepsilon_{e}=1 / 2$, the effective photon propagator of Eq. (8) has a square root branch cut

$$
\tilde{D}_{0}^{\mu \nu}(q)=\frac{1}{2} \frac{i}{\sqrt{-q^{2}}}\left(g^{\mu \nu}-\frac{\xi}{2} \frac{q^{\mu} q^{\nu}}{q^{2}}\right) .
$$

This momentum dependence is responsible for the appearance of Feynman diagrams with non-integer indices and is a major source of technical difficulty that we shall discuss in details in the next sections. As already noticed in Ref. [21, a similar momentum dependence can be found for $\mathrm{QED}_{3}$ in the large- $N_{F}$ limit where $N_{F}$ is the number of fermion species. The reason is the "infrared softening" of the photon propagator of $\mathrm{QED}_{3}$ for large $N_{F}$ 25126. Indeed, using a non-local gauge fixing term, $\eta$, as done in Ref. 27] and justified in the general case in Ref. 28, the dressed photon propagator together with the one-loop polarization operator of massless $\mathrm{QED}_{3}$, read:

$$
D^{\mu \nu}(q)=-i \frac{g^{\mu \nu}-\eta q^{\mu} q^{\nu} / q^{2}}{q^{2}\left[1-\Pi\left(q^{2}\right)\right]}, \quad \Pi_{1}\left(q^{2}\right)=-\frac{e^{2} N_{F}}{8 \sqrt{-q^{2}}},
$$

where, for $\eta=1$, the usual Landau gauge propagator is recovered, the lower index 1 on $\Pi$ refers to the order of perturbation theory and $e^{2}$ has dimension of mass in super-renormalizable $\mathrm{QED}_{3}$. In the $1 / N_{F}$ approximation $\left(N_{F} \rightarrow \infty\right.$ and $e^{2} \rightarrow 0$ with $e^{2} N_{F}$ fixed) and focusing on the infrared limit, $\sqrt{-q^{2}} \ll e^{2} N_{F}$, the dressed photon propagator of Eq. (11) becomes:

$$
D^{\mu \nu}(q)=\frac{8}{e^{2} N_{F}} \frac{i}{\sqrt{-q^{2}}}\left(g^{\mu \nu}-\eta \frac{q^{\mu} q^{\nu}}{q^{2}}\right),
$$

and, similarly to Eq. $\sqrt{10}$, behaves like $1 / \sqrt{-q^{2}}$ instead of the usual $1 / q^{2}$.

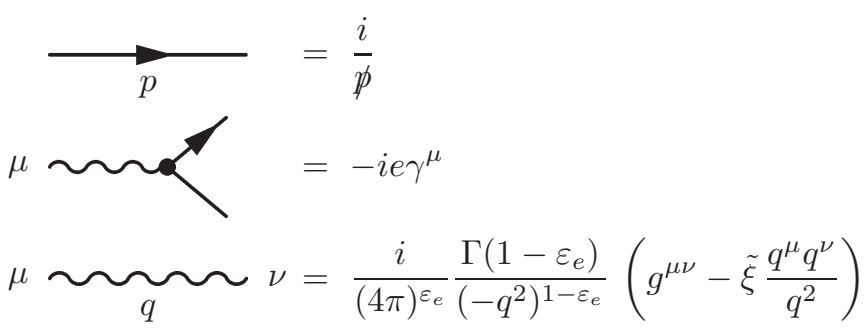

FIG. 1: Feynman rules for massless RQED.

Finally, the effective free photon propagator of $\operatorname{RQED}_{4,2}$, which has an exponent $\varepsilon_{e}=1$, has even a softer momentum dependence. The latter is similar to the momentum dependence of the dressed photon propagator of $\mathrm{QED}_{2}$ at large $N_{F}$. In the case of $\mathrm{RQED}_{4,2}$, however, the logarithmic divergence of the effective photon propagator translates into the appearance of a pole in the Gamma function of Eq. (8). This case requires some further regularization such as, e.g., giving a finite width to the 1-brane ${ }^{6}$. In the following, we shall mainly focus on the 2-brane case described by $\mathrm{RQED}_{4,3}$. The similarity between the reduced versions of QED and its large $N_{F}$ limit will be a subject of our future investigations.

With the Feynman rules of massless $\mathrm{RQED}_{d_{\gamma}, d_{e}}$ summarized on Fig. 1. perturbation theory is then implemented in the usual way by solving Dyson equations for the fermion and photon propagators and computing the various self-energies. Focusing for simplicity on the fermion propagator, which is central to the present work, the corresponding Dyson equation reads:

$$
S(p)=S_{0}(p)+S_{0}(p)(-i \Sigma(p)) S(p),
$$

where $\Sigma$ is the fermion self-energy. The solution to this equation in the case of massless fermions can be written as:

$$
-i \not p S(p)=\frac{1}{1-\Sigma_{V}\left(p^{2}\right)}, \quad \Sigma(p)=\not p \Sigma_{V}\left(p^{2}\right) .
$$

The effective divergence appearing in parentheses in Tab. I actually corresponds to the divergence of $\Sigma_{V}\left(p^{2}\right)$. Such a self-energy is divergent in all RQEDs as indicated in the table. The divergence is related to loop integrals depending on $d_{e}$. Following Refs. 20]21] we use dimensional regularization and work with an arbitrary $d_{e}$. The latter may be expressed as a function of the two epsilon parameters which naturally appeared at the level of Eq. (4). The parameter $\varepsilon_{e}$ takes into account the branelike nature of the system. For fixed $\varepsilon_{e}$ self-energies and related propagators take the form of Laurent series in $\varepsilon_{\gamma}$. Working in an improved minimal subtraction scheme $(\overline{\mathrm{MS}})$ we may then absorb the singular part of the series (in $\varepsilon_{\gamma}$ ) in renormalization constants which relate the bare fields and parameters of the theory to renormalized ones:

$$
\psi=Z_{\psi}^{1 / 2} \psi_{r}, \quad A=Z_{A}^{1 / 2} A_{r}, \quad a=Z_{A} a_{r}, \quad e=Z_{\alpha}^{1 / 2} e_{r},
$$


where $Z_{\psi}, Z_{A}$ and $Z_{\alpha}$ are the dimensionless renormalization constants. Moreover, in the $\overline{\mathrm{MS}}$ scheme we also define a dimensionless renormalized coupling constant $\alpha$ via the equation:

$$
\frac{\alpha(\mu)}{4 \pi}=\mu^{-2 \varepsilon_{\gamma}} \frac{e^{2}}{(4 \pi)^{d_{\gamma} / 2}} Z_{\alpha}^{-1}(\alpha(\mu)) e^{-\gamma_{E} \varepsilon_{\gamma}},
$$

where $\gamma_{E}$ is Euler's constant and the $\mu^{-2 \varepsilon_{\gamma}}$ factor compensates for the dimension of $e^{2}$. Equivalently, from Eq. (16), the bare coupling constant $e$ can be expressed via the renormalized coupling constant $\alpha(\mu)$.

From the above arguments, the relation between the bare and renormalized fermion propagators reads:

$$
S(p)=Z_{\psi}\left(\alpha(\mu), a_{r}(\mu)\right) S_{r}(p ; \mu),
$$

where all singularities are in $Z_{\psi}$ and $S_{r}$, the renormalized fermion propagator, is finite. Similarly, the relation between the bare and renormalized effective photon propagators reads:

$$
\tilde{D}^{\mu \nu}(q)=Z_{A}(\alpha(\mu)) \tilde{D}_{r}^{\mu \nu}(q ; \mu)
$$

where all singularities are in $Z_{A}$ and $\tilde{D}_{r}$, the renormalized effective photon propagator, is finite. Finally, one may introduce another constant, $Z_{\Gamma}$, for the vertex renormalization: $\Gamma^{\mu}=Z_{\Gamma} \Gamma_{r}^{\mu}$ where $\Gamma_{r}^{\mu}$ is finite. Because both the renormalized coupling and vertex are finite there is a constraint among the constants:

$$
Z_{\alpha}=\left(Z_{\Gamma} Z_{\psi}\right)^{-2} Z_{A}^{-1}
$$

Furthermore, as in usual QEDs, the Ward identity:

$$
Z_{\Gamma} Z_{\psi}=1
$$

is satisfied in a general RQED implying that coupling renormalization is entirely due to gauge field renormalization: $Z_{\alpha}=Z_{A}^{-1}$ [38 The beta function can then be expressed as a function of the anomalous scaling dimension of the gauge field as follows:

$$
\begin{aligned}
& \beta(\alpha(\mu))=\frac{d \log \alpha(\mu)}{d \log \mu}=-2 \varepsilon_{\gamma}+\gamma_{A}(\alpha(\mu)), \\
& \gamma_{A}(\alpha(\mu))=\frac{d \log Z_{A}(\alpha(\mu))}{d \log \mu} .
\end{aligned}
$$

These functions together with the renormalized photon propagator of RQED were considered in Refs. [2021] where it was shown, in accordance with dimensional analysis, that: $\beta(\alpha)=\gamma_{A}(\alpha)=0$ in $\operatorname{RQED}_{4, d_{e}}\left(d_{e}<4\right)$.

In the following we will compute the renormalization constant $Z_{\psi}$ and deduce the anomalous scaling dimension of the fermion field:

$$
\gamma_{\psi}\left(\alpha(\mu), a_{r}(\mu)\right)=\frac{d \log Z_{\psi}\left(\alpha(\mu), a_{r}(\mu)\right)}{d \log \mu} .
$$

Differentiating Eq. 17) with respect to $\mu$ and taking into account Eq. 22 as well as the fact that the bare propagator does not depend on the renormalization scale yields

$$
\frac{\mathrm{d} \log S_{r}(p ; \mu)}{\mathrm{d} \log \mu}=-\gamma_{\psi}\left(\alpha(\mu), a_{r}(\mu)\right)
$$

where $S_{r}$ depends on $\mu$ explicitly as well as through $\alpha$ and $a_{r}$. As will be shown later, the explicit $\mu$ dependence enters through the combination $-p^{2} / \mu^{2}$ and the renormalized propagator may be written:

$$
S_{r}(p ; \mu)=\frac{i}{\not p} s_{r}\left(\frac{-p^{2}}{\mu^{2}} ; \alpha ; a_{r}\right)
$$

In partial differential form the renormalization group equation, Eq. 23), may then be written as:

$$
\left(\frac{\partial}{\partial \log \mu}+\beta(\alpha) \frac{\partial}{\partial \log \alpha}-\gamma_{A}(\alpha) \frac{\partial}{\partial \log a_{r}}+\gamma_{\psi}\left(\alpha, a_{r}\right)\right) S_{r}(p ; \mu)=0
$$

Equivalently, we may use Eq. (24) in order to trade the partial derivative with respect to $\mu$ in Eq. (25) for a derivative with respect to momentum:

$$
\left(2 \frac{\partial}{\partial \log \frac{-p^{2}}{\mu^{2}}}-\beta(\alpha) \frac{\partial}{\partial \log \alpha}+\gamma_{A}(\alpha) \frac{\partial}{\partial \log a_{r}}-\gamma_{\psi}\left(\alpha, a_{r}\right)\right) s_{r}\left(\frac{-p^{2}}{\mu^{2}} ; \alpha ; a_{r}\right)=0 .
$$

We shall come back to the solution of this equation in a later section.

\section{One-loop results}

Having set the basic notations and goals, we summarize here the one-loop results obtained in Ref. 20. As will be seen below, all diagrams can be compactly expressed as a function of the one-loop massless propagator diagram 


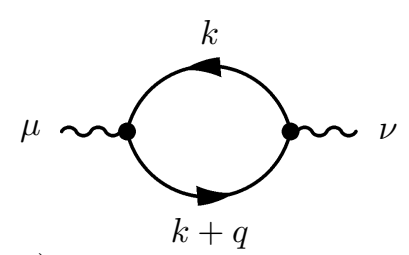

a)

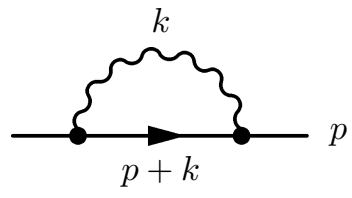

b)

FIG. 2: One-loop diagrams: a) photon self-energy and b) fermion self-energy.

defined as:

$$
\int \frac{\mathrm{d}^{d_{e}} k}{\left[-(k+p)^{2}\right]^{\alpha}\left[-k^{2}\right]^{\beta}}=\frac{i \pi^{d_{e} / 2}}{\left(-p^{2}\right)^{\alpha+\beta-d_{e} / 2}} G(\alpha, \beta),
$$

where $\alpha$ and $\beta$ are arbitrary indices labeling the diagram, the power of $-p^{2}$ follows from dimensional analysis and $G(\alpha, \beta)$ is the (dimensionless) coefficient function associated with the diagram. The latter is known exactly in the one-loop case:

$$
G(\alpha, \beta)=\frac{a(\alpha) a(\beta)}{a\left(\alpha+\beta-d_{e} / 2\right)} \quad a(\alpha)=\frac{\Gamma\left(d_{e} / 2-\alpha\right)}{\Gamma(\alpha)} .
$$

The one-loop diagrams, see Fig. 2, are defined by:

$$
\begin{aligned}
& i \Pi_{1}^{\mu \nu}(q)=-\int \frac{\mathrm{d}^{d_{e}} k}{(2 \pi)^{d_{e}}} \operatorname{Tr}\left[\left(-i e \gamma^{\mu}\right) \frac{i(\not k+\not q)}{(k+q)^{2}}\left(-i e \gamma^{\nu}\right) \frac{i k}{k^{2}}\right], \\
& -i \Sigma_{1}(p)=\int \frac{\mathrm{d}^{d_{e}} k}{(2 \pi)^{d_{e}}}\left(-i e \gamma^{\mu}\right) \frac{i(\not p+\not k)}{(p+k)^{2}}\left(-i e \gamma^{\nu}\right) \frac{i}{(4 \pi)^{\varepsilon_{e}}} \frac{\Gamma\left(1-\varepsilon_{e}\right)}{\left(-k^{2}\right)^{1-\varepsilon_{e}}}\left(g_{\mu \nu}-\tilde{\xi} \frac{k_{\mu} k_{\nu}}{k^{2}}\right),
\end{aligned}
$$

where the lower index 1 on $\Pi$ and $\Sigma$ refers to one-loop, $\not k=\gamma^{\mu} k_{\mu}, k^{2}=k^{\mu} k_{\mu}, g^{\mu \nu}=\operatorname{diag}(1,-1,-1, \cdots,-1)$ is the metric tensor in the $d_{e}$-dimensional space-time and $\operatorname{Tr}$ denotes the trace in gamma-matrix space. The computation of the above integrals first involves gamma-matrix algebra at the level of the numerators in Eqs. (29). The following trace and contraction identities summarize the numerator algebra of RQED:

$$
\begin{aligned}
& \left\{\gamma^{\mu}, \gamma^{\nu}\right\}=2 g^{\mu \nu}, \quad \operatorname{Tr}[\mathbb{1}]=d, \quad \operatorname{Tr}\left[\text { odd number of } \gamma^{\prime} \mathrm{s}\right]=0, \quad \operatorname{Tr}\left[\gamma^{\mu} \gamma^{\nu}\right]=d g^{\mu \nu}, \quad \operatorname{Tr}\left[\gamma^{\mu} \gamma^{\alpha} \gamma_{\mu} \gamma^{\beta}\right]=-d\left(d_{e}-2\right) g^{\alpha \beta}, \\
& \operatorname{Tr}\left[\gamma_{\mu} \gamma^{\alpha} \gamma_{\nu} \gamma^{\beta} \gamma^{\mu} \gamma^{\delta} \gamma^{\nu} \gamma^{\theta}\right]=d g^{\alpha \beta} g^{\delta \theta}\left(4-d_{e}\right)\left(d_{e}-2\right)-d g^{\alpha \delta} g^{\beta \theta}\left(8-d_{e}\right)\left(d_{e}-2\right)+d g^{\alpha \theta} g^{\delta \beta}\left(4-d_{e}\right)\left(d_{e}-2\right), \\
& \gamma^{\mu} \gamma_{\mu}=g^{\mu}{ }_{\mu}=d_{e}, \quad \gamma^{\mu} \gamma^{\alpha} \gamma_{\mu}=-\left(d_{e}-2\right) \gamma^{\alpha}, \quad \gamma^{\mu} \gamma^{\alpha} \gamma^{\beta} \gamma_{\mu}=2 \gamma^{\beta} \gamma^{\alpha}+\left(d_{e}-2\right) \gamma^{\alpha} \gamma^{\beta}, \\
& \gamma^{\mu} \gamma^{\alpha} \gamma^{\beta} \gamma^{\delta} \gamma_{\mu}=-2 \gamma^{\delta} \gamma^{\beta} \gamma^{\alpha}-\left(d_{e}-4\right) \gamma^{\alpha} \gamma^{\beta} \gamma^{\delta},
\end{aligned}
$$

where we have used $d$-dimensional fermion spinors in such a way that the gamma matrices have dimension $d \times d$. Alternatively, we may use the number of massless fermion fields, $N_{F}$, which is related to $d$ via:

$$
N_{F}=\frac{d}{4}
$$

in such a way that a single fermion specie is represented by a four-dimensional spinor such as in usual $\mathrm{QED}_{4}$. In the following, we shall work either with an arbitrary $d$ for the sake of generality or, equivalently, with $N_{F}$ to relate our formulas to well known expressions in $\mathrm{QED}_{4}$ and $\mathrm{QED}_{3}$ with $N_{F}$ fermion species. A further simplification of Eq. (29b) comes from combining the expression of $\Sigma_{V}$ in Eq. (14) with the trace identities of Eq. (30) in such a way that:

$$
\Sigma_{V}\left(p^{2}\right)=\frac{-1}{d\left(-p^{2}\right)} \operatorname{Tr}[\not p \Sigma(p)] .
$$

We may proceed in a similar way for the polarization operator in Eq. 29a. Because of current conservation the latter is constrained to have the form:

$$
\Pi^{\mu \nu}(q)=\left(g^{\mu \nu} q^{2}-q^{\mu} q^{\nu}\right) \Pi\left(q^{2}\right), \quad \Pi\left(q^{2}\right)=\frac{-\Pi^{\mu}{ }_{\mu}(q)}{\left(d_{e}-1\right)\left(-q^{2}\right)} .
$$




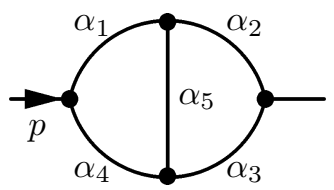

FIG. 3: Two-loop massless propagator diagram.

and the effective divergence appearing in parentheses in Tab. I actually corresponds to the divergence of $\Pi\left(q^{2}\right)$.

With the above conventions and identities in hand, Eqs. 29. can be straightforwardly computed in an arbitrary gauge. The final result reads:

$$
\begin{aligned}
& \Pi_{1}\left(q^{2}\right)=-d \frac{e^{2}}{(4 \pi)^{d_{e} / 2}\left(-q^{2}\right)^{\varepsilon_{\gamma}+\varepsilon_{e}}} \frac{d_{e}-2}{2\left(d_{e}-1\right)} G(1,1), \\
& \Sigma_{V 1}\left(p^{2}\right)=-\frac{e^{2} \Gamma\left(1-\varepsilon_{e}\right)}{(4 \pi)^{d_{\gamma} / 2}\left(-p^{2}\right)^{\varepsilon_{\gamma}}} \frac{d_{e}-2}{2}\left[\frac{2\left(d_{e}-2\right)}{d_{\gamma}+d_{e}-4}-\xi\right] G\left(1,1-\varepsilon_{e}\right) .
\end{aligned}
$$

As can be seen from Eqs. (34), the one-loop results are expressed in terms of two master integrals: $G(1,1)$ for the photon self-energy and $G\left(1,1-\varepsilon_{e}\right)$ for the fermion self-energy where $G$ is given by Eq. (28). These equations have been discussed in Ref. [20] and we refer the interested reader to this reference for more details. These equations will be used in the next sections concerning the computation of the two-loop fermion self-energy and renormalization.

\section{TWO-LOOP FERMION SELF-ENERGY}

\section{A. The two-loop massless propagator diagram}

In the one-loop case, after working out the numerator algebra, the considered diagrams could be expressed in terms of two simple scalar integrals $G(1,1)$ and $G\left(1,1-\varepsilon_{e}\right)$, see Eqs. (34). These one-loop integrals are known exactly from Eq. (28). At the two-loop level the situation is more complicated. The two-loop massless propagator diagram is actually one of the basic building blocks of multi-loop calculations and has a long history, see the review Ref. 29. For arbitrary indices $\alpha_{i}$ and external momentum $p$ in a Minkowski space-time of dimensionality $d_{e}$ the two-loop massless propagator diagram, see Fig. 3 , reads:

$$
\iint \frac{\mathrm{d}^{d_{e}} k_{1} \mathrm{~d}^{d_{e}} k_{2}}{\left[-\left(k_{1}+p\right)^{2}\right]^{\alpha_{1}}\left[-\left(k_{2}+p\right)^{2}\right]^{\alpha_{2}}\left[-k_{2}^{2}\right]^{\alpha_{3}}\left[-k_{1}^{2}\right]^{\alpha_{4}}\left[-\left(k_{2}-k_{1}\right)^{2}\right]^{\alpha_{5}}}=-\frac{\pi^{d_{e}}}{\left(-p^{2}\right)^{\sum_{i=1}^{5} \alpha_{i}-d_{e}}} G\left(\alpha_{1}, \alpha_{2}, \alpha_{3}, \alpha_{4}, \alpha_{5}\right),
$$

where, on the right-hand side, the power of $p$ follows from dimensionality and the function $G\left(\left\{\alpha_{i}\right\}\right)$ is the 2-loop (dimensionless) coefficient function of the diagram. One of the main goals of multi-loop calculations is an exact evaluation of $G\left(\left\{\alpha_{i}\right\}\right)$.

When all indices are integers this function can be computed exactly using IBP identities $\frac{14115}{15}$ The diagram can then be expressed in terms of recursively one-loop integrals as can be seen from the following well-known example ${ }^{39}$

$$
G(1,1,1,1,1)=C_{D}[\overbrace{1}^{1} 1=\frac{2}{d_{e}-4}\left[\left(3-d_{e}\right) G^{2}(1,1)+\left(9\left(d_{e}-2\right)+\frac{8}{d_{e}-4}\right) G(1,1) G\left(1, \varepsilon_{e}+\varepsilon_{\gamma}\right)\right],
$$

where $C_{D}$ denotes the coefficient function of the diagram.

For arbitrary indices its evaluation is however highly nontrivial: it can be represented ${ }^{30}$ as a combination of twofold series. In some simpler cases explicit analytical results can be obtained!13/14]18/21]31]34 As an example, the diagram $G(1,1,1,1, \alpha)$ can be expressed in a compact way for a special value of the non-integer index on the central line. $\frac{142132}{121}$

$$
G\left(1,1,1,1, \lambda_{e}\right)=C_{D}\left[\lambda_{1}^{1} \lambda_{e}\right]=3 \frac{\Gamma\left(\lambda_{e}\right) \Gamma\left(1-\lambda_{e}\right)}{\Gamma\left(2 \lambda_{e}\right)}\left[\Psi_{2}\left(\lambda_{e}\right)-\Psi_{2}(1)\right], \quad\left(\lambda_{e}=d_{e} / 2-1\right)
$$

where $\Psi_{2}(x)=\Psi_{1}^{\prime}(x)$ is the trigamma function and $\Psi_{1}(x)$ is the digamma function. The result of Eq. (37) elegantly covers the cases of $\mathrm{RQED}_{4, d_{e}}$ 's where $\varepsilon_{\gamma} \rightarrow 0$ and in particular $\mathrm{RQED}_{4,3}$ where the gauge field does not renormalize. 


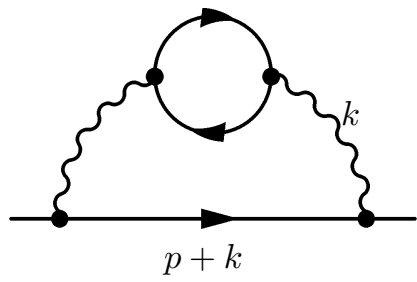

a)

b)

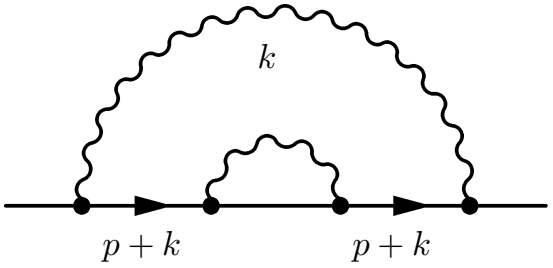

c)

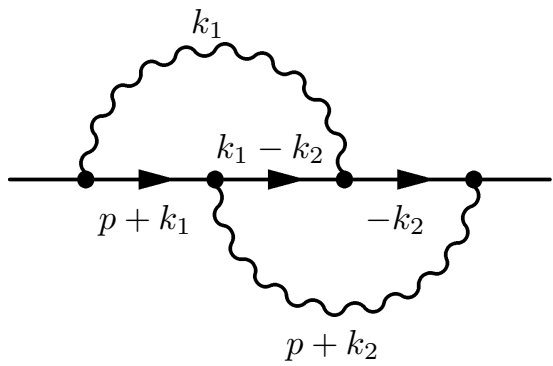

FIG. 4: 2-loop fermion self-energy diagrams: a) bubble diagram, b) rainbow diagram and c) crossed photon diagram.

In the general case of $\mathrm{RQED}_{d_{\gamma}, d_{e}}$ such a diagram appears in the calculation of the two-loop photon self-energy but with a more general index: $\alpha=1-\varepsilon_{e}=\lambda_{e}+\varepsilon_{\gamma}$ where $\varepsilon_{\gamma}$ can be non-zero. In this case, the result is more complicated and reads $\underline{13}$

$$
\begin{aligned}
G(1,1,1,1, \alpha) & =C_{D}[\alpha)_{1}^{1}=-2 \Gamma\left(\lambda_{e}\right) \Gamma\left(\lambda_{e}-\alpha\right) \Gamma\left(1-2 \lambda_{e}+\alpha\right) \times \\
& \times\left[\frac{\Gamma\left(\lambda_{e}\right)}{\Gamma\left(2 \lambda_{e}\right) \Gamma\left(3 \lambda_{e}-\alpha-1\right)} \sum_{n=0}^{\infty} \frac{\Gamma\left(n+2 \lambda_{e}\right) \Gamma(n+1)}{n ! \Gamma(n+1+\alpha)} \frac{1}{n+1-\lambda_{e}+\alpha}+\frac{\pi \cot \pi\left(2 \lambda_{e}-\alpha\right)}{\Gamma\left(2 \lambda_{e}\right)}\right],
\end{aligned}
$$

where the one-fold series corresponds to a generalized hypergeometric function, ${ }_{3} F_{2}$, of argument 1 . The diagram evaluated in Eq. (38) is one of the simplest among a class of complicated diagrams that have been considered in Ref. [13] on the basis of a new development of the Gegenbauer polynomial technique. This class includes diagrams with two adjacent lines having integer indices while three other lines have arbitrary indices; the corresponding coefficient function is given by: $G(\alpha, 1, \beta, \gamma, 1)$. As proved in Ref. [13] all of these diagrams may be expressed in terms of generalized hypergeometric functions ${ }_{3} F_{2}$ of argument 1 . For this class of diagrams, similar results have been found in Ref. [33] using an ansatz to solve the recurrence relations for the two-loop diagram. However, beyond the simplest case with a single non-integer index represented by Eq. (38), explicit expressions for other diagrams of this class are unknown.

As will be seen below, it turns out that the computation of the two-loop fermion self-energy for a general RQED $d_{\gamma}, d_{e}$ requires the knowledge of a two-loop massless propagator diagram with two non-integer indices on non-adjacent lines:

$$
G(\alpha, 1, \beta, 1,1)=C_{D}[\overbrace{1}^{\alpha} 1
$$

Using the results of Ref. [13] an exact explicit expression for this diagram can be derived, see App. B for general formulas. As can be seen from the latter, the result is of higher complexity than the one of Eq. (38). For the sake of simplicity we shall not consider the general solution here. Instead, and in the spirit of Ref. 21], we shall focus on the less general but important case of $\mathrm{RQED}_{4, d_{e}}$ 's for which $\varepsilon_{\gamma} \rightarrow 0$. As will be shown below, in this case a simpler solution can be found using integration by parts.

\section{B. Reduction to master integrals}

Having set all the perturbative framework in Sec. II and presented the two-loop massless propagator diagram in Sec. III A, we now proceed on computing the two-loop fermion self-energy. The latter consists of three diagrams that are displayed on Fig. 4.

The first diagram, Fig. 4a , is the so-called bubble diagram which is defined as:

$$
\begin{aligned}
& -i \Sigma_{2 a}(p)= \\
& \int \frac{\mathrm{d}^{d_{e}} k}{(2 \pi)^{d_{e}}}\left(-i e \gamma^{\alpha}\right) \frac{i(\not p+\not k)}{(p+k)^{2}}\left(-i e \gamma^{\beta}\right) \frac{i}{(4 \pi)^{\varepsilon_{e}}} \frac{\Gamma\left(1-\varepsilon_{e}\right)}{\left(-k^{2}\right)^{1-\varepsilon_{e}}}\left(g_{\alpha \mu}-\tilde{\xi} \frac{k_{\alpha} k_{\mu}}{k^{2}}\right) i \Pi_{1}^{\mu \nu}(k) \frac{i}{(4 \pi)^{\varepsilon_{e}}} \frac{\Gamma\left(1-\varepsilon_{e}\right)}{\left(-k^{2}\right)^{1-\varepsilon_{e}}}\left(g_{\nu \beta}-\tilde{\xi} \frac{k_{\nu} k_{\beta}}{k^{2}}\right),
\end{aligned}
$$


where the lower index 2 in $\Sigma_{2 a}$ refers to 2-loop and $\Pi_{1}^{\mu \nu}(k)$ is a one-loop photon self-energy insertion corresponding to Eq. (34a). This diagram is obviously gauge invariant. This follows from current conservation which implies that $k_{\mu} \Pi^{\mu \nu}=0$ so that all terms proportional to the gauge fixing term vanish. Using Eq. (32) and performing all the numerator algebra with the help of Eqs. 30 yields:

$$
\Sigma_{V 2 a}\left(p^{2}\right)=d \frac{e^{4} \Gamma^{2}\left(1-\varepsilon_{e}\right)}{(4 \pi)^{d_{\gamma}}\left(-p^{2}\right)^{2 \varepsilon_{\gamma}}} \frac{\left(d_{e}-2\right)^{2}}{2\left(2 d_{\gamma}-d_{e}-6\right)} G(1,1) G\left(1, \varepsilon_{\gamma}-\varepsilon_{e}\right)
$$

where the factor of $d$ is due to the fermion loop and the master integral, $G(1,1) G\left(1, \varepsilon_{\gamma}-\varepsilon_{e}\right)$, is a simple product of two one-loop integrals which can be straightforwardly evaluated with the help of Eq. (28).

The second diagram, Fig. $4 \mathrm{~b}$, is the so-called rainbow diagram which is defined as:

$$
-i \Sigma_{2 b}(p)=\int \frac{\mathrm{d}^{d_{e}} k}{(2 \pi)^{d_{e}}}\left(-i e \gamma^{\mu}\right) \frac{i(\not p+\not k)}{(p+k)^{2}}\left(-i \Sigma_{1}(p+k)\right) \frac{i(\not p+\not k)}{(p+k)^{2}}\left(-i e \gamma^{\nu}\right) \frac{i}{(4 \pi)^{\varepsilon_{e}}} \frac{\Gamma\left(1-\varepsilon_{e}\right)}{\left(-k^{2}\right)^{1-\varepsilon_{e}}}\left(g_{\mu \nu}-\tilde{\xi} \frac{k_{\mu} k_{\nu}}{k^{2}}\right)
$$

where $\Sigma_{1}(k)=\not k \Sigma_{V 1}\left(k^{2}\right)$ is a one-loop fermion self-energy insertion corresponding to Eq. (34b). This diagram is not gauge invariant. Proceeding along the same lines as for the bubble diagram yields:

$$
\Sigma_{V 2 b}\left(p^{2}\right)=\frac{e^{4} \Gamma^{2}\left(1-\varepsilon_{e}\right)}{(4 \pi)^{d_{\gamma}}\left(-p^{2}\right)^{2 \varepsilon_{\gamma}}} \frac{\left(d_{e}-2\right)\left(d_{\gamma}-3\right)\left(d_{\gamma}+d_{e}-4\right)}{2\left(d_{\gamma}-4\right)}\left(\frac{2\left(d_{e}-2\right)}{d_{\gamma}+d_{e}-4}-\xi\right)^{2} G\left(1,1-\varepsilon_{e}\right) G\left(1-\varepsilon_{e}, \varepsilon_{\gamma}\right)
$$

where the master integral, $G\left(1,1-\varepsilon_{e}\right) G\left(1-\varepsilon_{e}, \varepsilon_{\gamma}\right)$, is also a simple product of two one-loop integrals which can be straightforwardly evaluated with the help of Eq. (28).

Finally, the third diagram, Fig. 4, which is the so-called crossed photon diagram is a truly two-loop diagram. It is defined as:

$$
\begin{aligned}
-i \Sigma_{2 c}(p)= & \int \frac{\mathrm{d}^{d_{e}} k_{1} \mathrm{~d}^{d_{e}} k_{2}}{(2 \pi)^{2 d_{e}}}\left(-i e \gamma^{\mu}\right) \frac{-i \not k_{2}}{k_{2}^{2}} \frac{i}{(4 \pi)^{\varepsilon_{e}}} \frac{\Gamma\left(1-\varepsilon_{e}\right)}{\left(-\left(p+k_{2}\right)^{2}\right)^{1-\varepsilon_{e}}}\left(g_{\mu \beta}-\tilde{\xi} \frac{\left(p+k_{2}\right)_{\mu}\left(p+k_{2}\right)_{\beta}}{\left(p+k_{2}\right)^{2}}\right) \times \\
& \times\left(-i e \gamma^{\alpha}\right) \frac{i\left(\not k_{1}-\not k_{2}\right)}{\left(k_{1}-k_{2}\right)^{2}} \frac{i}{(4 \pi)^{\varepsilon_{e}}} \frac{\Gamma\left(1-\varepsilon_{e}\right)}{\left(-k_{1}^{2}\right)^{1-\varepsilon_{e}}}\left(g_{\alpha \nu}-\tilde{\xi} \frac{k_{1 \alpha} k_{1 \nu}}{k_{1}^{2}}\right)\left(-i e \gamma^{\beta}\right) \frac{i\left(\not p+\not k_{1}\right)}{\left(p+k_{1}\right)^{2}}\left(-i e \gamma^{\nu}\right) .
\end{aligned}
$$

Proceeding along the same lines as for the two previous diagrams yields:

$$
\begin{aligned}
\Sigma_{V 2 c}\left(p^{2}\right)= & -\frac{e^{4} \Gamma^{2}\left(1-\varepsilon_{e}\right)}{(4 \pi)^{d_{\gamma}\left(-p^{2}\right)^{2 \varepsilon_{\gamma}}} \frac{d_{e}-2}{2}} \\
\times & \left\{\left(d_{e}-4-4 \frac{d_{\gamma}-d_{e}}{d_{\gamma}+d_{e}-4}-2 \frac{\left(d_{e}-2\right)^{2}}{d_{\gamma}+d_{e}-4} \xi+\frac{d_{e}-2}{2} \xi^{2}\right) G^{2}\left(1,1-\varepsilon_{e}\right)+\right. \\
& +\left(d_{e}+4+8 \frac{d_{e}-2}{d_{\gamma}+d_{e}-4}+\frac{16}{d_{\gamma}-4}+4 \frac{\left(d_{e}-2\right)\left(d_{\gamma}-3\right)}{d_{\gamma}+d_{e}-4} \xi-\left(d_{\gamma}-3\right) \xi^{2}\right) G\left(1,1-\varepsilon_{e}\right) G\left(1-\varepsilon_{e}, \varepsilon_{\gamma}\right)+ \\
& \left.+4 G\left(1-\varepsilon_{e}, 1,-1-\varepsilon_{e}, 1,1\right)-4 G\left(1-\varepsilon_{e}, 1,-\varepsilon_{e}, 1,1\right)+\left(8-d_{e}\right) G\left(-\varepsilon_{e}, 1,-\varepsilon_{e}, 1,1\right)\right\}
\end{aligned}
$$

where, besides the primitively one-loop master integrals, $G^{2}\left(1,1-\varepsilon_{e}\right)$ and $G\left(1,1-\varepsilon_{e}\right) G\left(1-\varepsilon_{e}, \varepsilon_{\gamma}\right)$, complicated two-loop propagator diagrams of the type of Eq. (39) enter the expression of the self-energy. It is interesting to notice that none of the complicated terms depend on the gauge parameter, $\xi$. That is, working in the Feynman gauge, $\xi=0$, simplifies the calculations but does not reduce the complexity of the diagrams.

\section{Integration by parts and self-energy of the crossed photon diagram}

In order to further reduce the complicated two-loop diagrams appearing in Eq. 45 we use an IBP identity which follows from the homogeneity of the two-loop propagator diagram, Eq. (35), in $p$, see Ref. 15] and Ref. [11 for a 
review. In graphical form, this IBP reads:

$$
\left(\frac{d_{e}}{2}+\alpha_{4}-\alpha_{1}-\alpha_{2}-\alpha_{5}\right)
$$

where \pm on the right-hand side of the equation denotes the increase or decrease of a line index by 1 with respect to its value on the left-hand side. This expression slightly simplifies in the cases we are interested in:

$$
\begin{aligned}
& \left(1-\varepsilon_{e}-\varepsilon_{\gamma}-\alpha\right) \overbrace{\beta}^{\alpha}=\beta(2>1 \\
& +\left(3-3 \varepsilon_{e}-3 \varepsilon_{\gamma}-\alpha-\beta\right)\left(-p^{2}\right)^{-1}\left(1 c_{\beta}^{\alpha-1}\right.
\end{aligned}
$$

where we have used Eq. (5) and the fact that, in $p$-space, a line with zero index shrinks to a point. As a consequence, on the right-hand side of Eq. (47) all diagrams except the third one are recursively one-loop diagram.

In order to see how Eq. (47) can be usefully implemented, let's consider the case where $\alpha=\beta=1-\varepsilon_{e}$. In this case Eq. 47) reduces to:

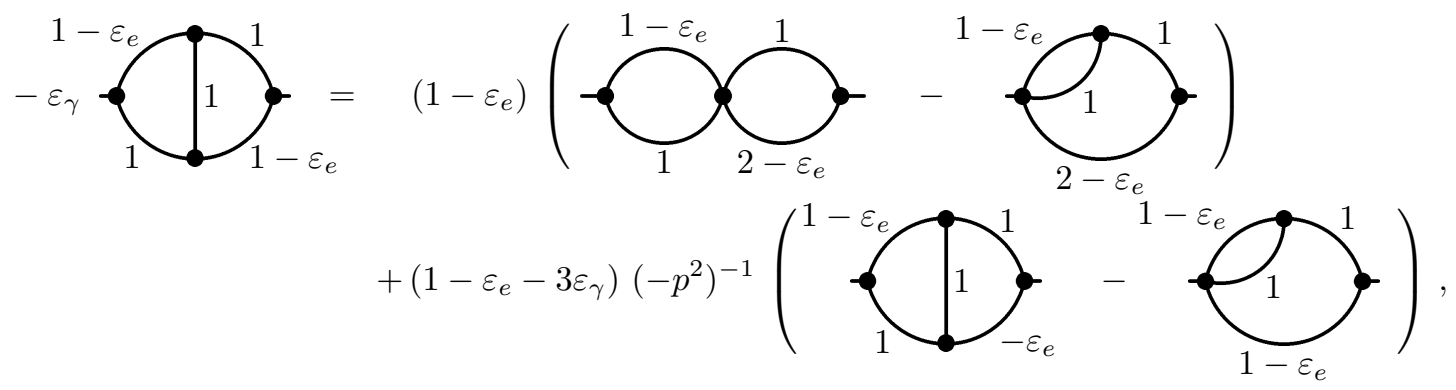

where we have used the symmetry of the two-loop diagram: $G(\alpha, 1, \beta, 1,1)=G(\beta, 1, \alpha, 1,1)$. We then see that the truly two-loop diagram appearing on the right-hand side of Eq. (48) has a coefficient function $G\left(1-\varepsilon_{e}, 1,-\varepsilon_{e}, 1,1\right)$. It is therefore one of the complicated diagrams appearing in Eq. 45 that we would like to compute. At this point, it is important to notice that the function $G\left(1-\varepsilon_{e}, 1,-\varepsilon_{e}, 1,1\right)$ is associated with an ultra-violet singular two-loop diagram in the limit $\varepsilon_{\gamma} \rightarrow 0$. Indeed, power counting shows that this diagram is proportional to: $\left(-p^{2}\right)^{-2 \varepsilon_{\gamma}}$, i.e., diverges logarithmically. On the other-hand, the two-loop diagram appearing on the left-hand side of Eq. [48], the coefficient function of which is given by: $G\left(1-\varepsilon_{e}, 1,1-\varepsilon_{e}, 1,1\right)$, is proportional to $\left(-p^{2}\right)^{-1-2 \varepsilon_{\gamma}}$; it is therefore ultra-violet convergent in the limit $\varepsilon_{\gamma} \rightarrow 0$. As a consequence, the Laurent series associated to its $\varepsilon_{\gamma}$-expansion reduces to the regular part:

$$
G\left(1-\varepsilon_{e}, 1,1-\varepsilon_{e}, 1,1\right)=\sum_{n=0}^{\infty} c_{n}\left(\varepsilon_{e}\right) \varepsilon_{\gamma}^{n}
$$

For $\varepsilon_{e}=0$, which applies to $\mathrm{QED}_{4}$ and $\mathrm{QED}_{3}$, this diagram corresponds to the well-known Eq. (36). On the other hand, for an arbitrary non-integer $\varepsilon_{e}$, for example $\varepsilon_{e}=1 / 2$ in the case of $\operatorname{RQED}_{4,3}$, it belongs to the class of complicated diagrams, see Eq. (39) and related discussion around this equation, whose explicit analytic expression, and hence the coefficients $c_{n}$, is presently unknown. However, the fact that this diagram is convergent together with its coefficient $\varepsilon_{\gamma}$, implies that the left-hand side of Eq. (48) vanishes in the limit $\varepsilon_{\gamma} \rightarrow 0$. Hence, from the right-hand 
side of Eq. (48) we find that:

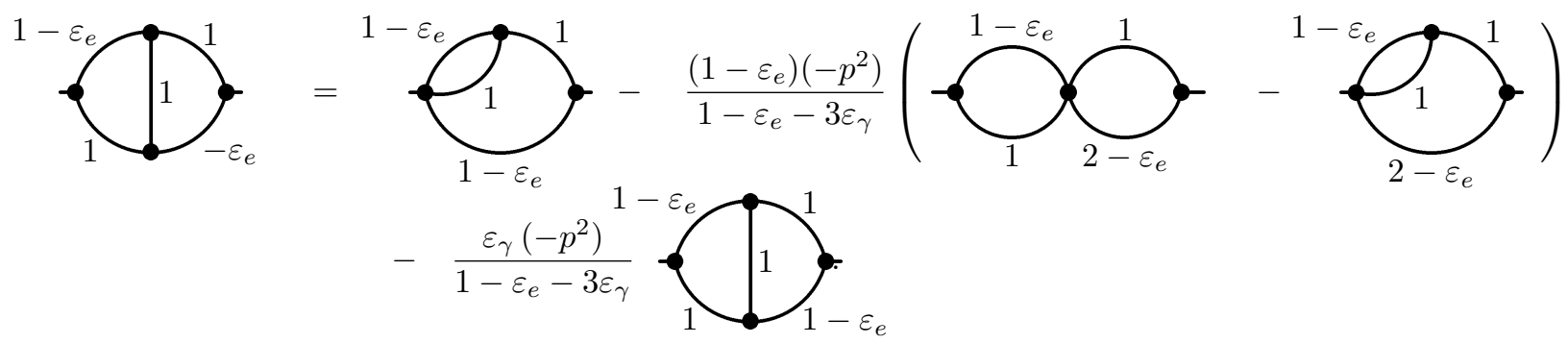

After some algebra, the recursively one-loop diagrams appearing in the left-hand side of Eq. $(50)$ can be expressed in terms of the one-loop master integrals entering the expression of the fermion self-energy Eq. (45). The result reads:

$$
\begin{aligned}
G\left(1-\varepsilon_{e}, 1,-\varepsilon_{e}, 1,1\right) & =-2 \frac{\left(d_{\gamma}-3\right)\left(2 d_{\gamma}+d_{e}-8\right)}{\left(d_{\gamma}-4\right)\left(d_{\gamma}+d_{e}-6\right)} G\left(1,1-\varepsilon_{e}\right) G\left(1-\varepsilon_{e}, \varepsilon_{\gamma}\right)+\frac{d_{\gamma}+d_{e}-6}{2 d_{\gamma}+d_{e}-10} G^{2}\left(1,1-\varepsilon_{e}\right) \\
& +\frac{d_{\gamma}-4}{2 d_{\gamma}+d_{e}-10} G\left(1-\varepsilon_{e}, 1,1-\varepsilon_{e}, 1,1\right)
\end{aligned}
$$

where the last term does not contribute in the limit $d_{\gamma} \rightarrow 4$. Hence, for $\mathrm{RQED}_{4, d_{e}}$ and in particular in the case of $\mathrm{RQED}_{4,3}$ where the last diagram in Eq. (51) is non-trivial, the function $G\left(1-\varepsilon_{e}, 1,-\varepsilon_{e}, 1,1\right)$ is known for arbitrary $\varepsilon_{e}$ to $\mathrm{O}\left(\varepsilon_{\gamma}\right)$. The knowledge of these lowest order terms is enough, e.g., to compute the anomalous scaling dimension of the fermion field at two-loop (see below for more).

We may proceed in a similar way for the two other complicated diagrams appearing in Eq. (45). The corresponding coefficient functions, $G\left(1-\varepsilon_{e}, 1,-1-\varepsilon_{e}, 1,1\right)$ and $G\left(-\varepsilon_{e}, 1,-\varepsilon_{e}, 1,1\right)$, are proportional to $\left(-p^{2}\right)^{1-2 \varepsilon_{\gamma}}$ and are strongly UV divergent. They can both be expressed in terms of $G\left(1-\varepsilon_{e}, 1,-\varepsilon_{e}, 1,1\right)$. In order to see this, we consider the case where $\alpha=-\varepsilon_{e}$ and $\beta=1-\varepsilon_{e}$ for which Eq. (47) reduces to:

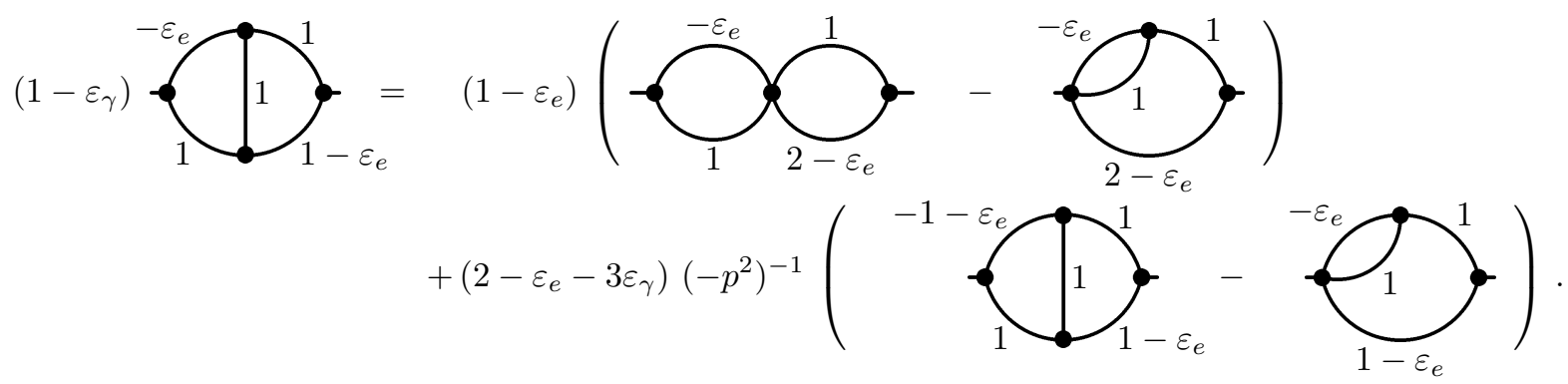

Eq. (52) shows that there is a simple relation, i.e., involving recursively one-loop diagrams, between two of the complicated diagrams, $G\left(1-\varepsilon_{e}, 1,-1-\varepsilon_{e}, 1,1\right)$ and $G\left(1-\varepsilon_{e}, 1,-\varepsilon_{e}, 1,1\right)$, entering the fermion self-energy, Eq. (45). Expressing the, still unknown, coefficient function $G\left(1-\varepsilon_{e}, 1,-1-\varepsilon_{e}, 1,1\right)$ in terms of $G\left(1-\varepsilon_{e}, 1,-\varepsilon_{e}, 1,1\right)$ that we have computed above, see Eq. (51), yields, in graphical form:

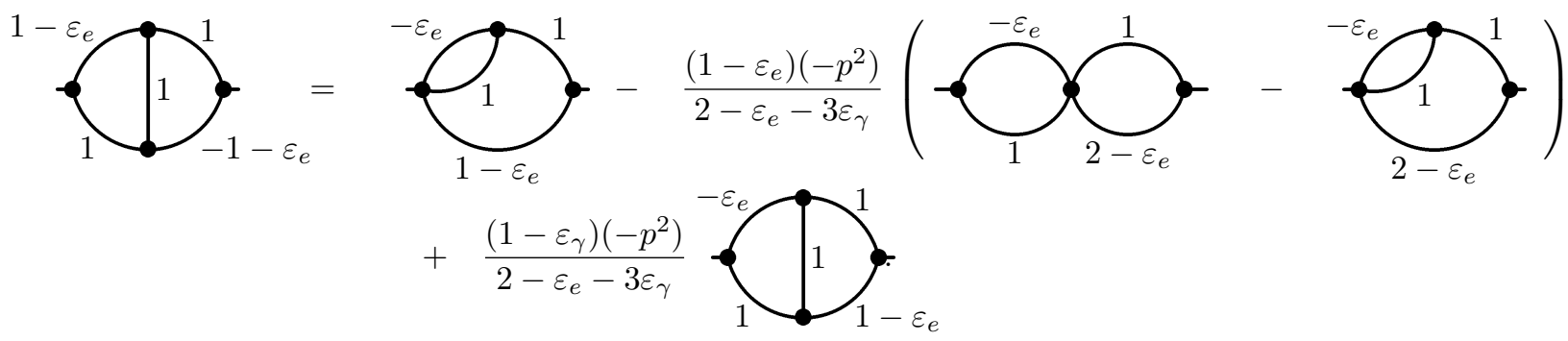

After some algebra, the recursively one-loop diagrams appearing in the right-hand side of Eq. (53) can also be expressed in terms of the one-loop master integrals that enter the expression of the fermion self-energy Eq. 45. Together with 
Eq. (51), the final result reads:

$$
\begin{aligned}
G\left(1-\varepsilon_{e}, 1,-1-\varepsilon_{e}, 1,1\right) & =-\frac{d_{\gamma}-2}{d_{\gamma}-4}\left(\frac{2\left(d_{\gamma}-3\right)}{d_{\gamma}+d_{e}-6}+\frac{d_{\gamma}-d_{e}}{d_{\gamma}+d_{e}-4}\right) G\left(1,1-\varepsilon_{e}\right) G\left(1-\varepsilon_{e}, \varepsilon_{\gamma}\right) \\
& +\frac{d_{\gamma}+d_{e}-6}{2 d_{\gamma}+d_{e}-8}\left(\frac{d_{\gamma}-2}{2 d_{\gamma}+d_{e}-10}+\frac{d_{\gamma}-d_{e}}{d_{\gamma}+d_{e}-4}\right) G^{2}\left(1,1-\varepsilon_{e}\right) \\
& +\frac{\left(d_{\gamma}-2\right)\left(d_{\gamma}-4\right)}{\left(2 d_{\gamma}+d_{e}-10\right)\left(2 d_{\gamma}+d_{e}-8\right)} G\left(1-\varepsilon_{e}, 1,1-\varepsilon_{e}, 1,1\right) .
\end{aligned}
$$

Finally, let's consider the case where $\alpha=1-\varepsilon_{e}$ and $\beta=-\varepsilon_{e}$ for which Eq. 477 reduces to:

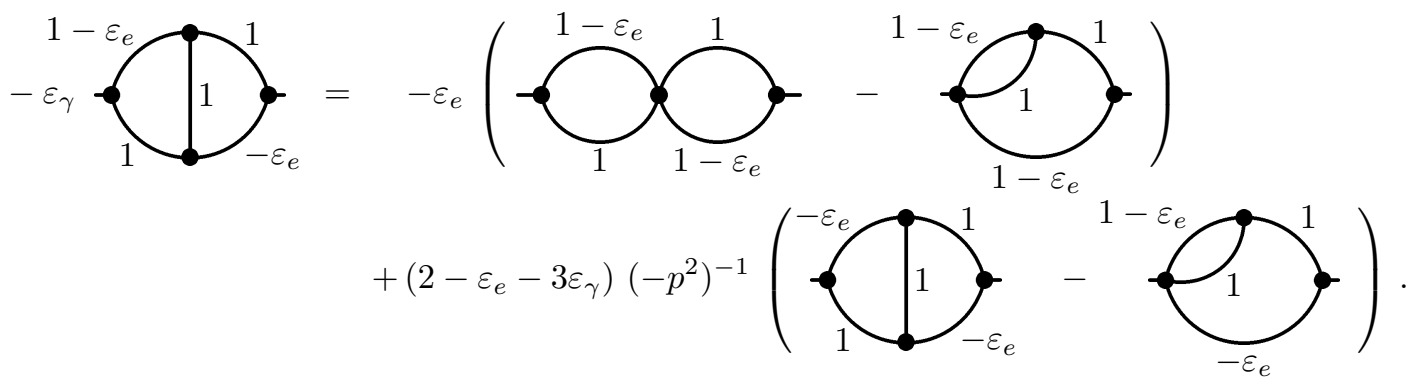

From Eq. (55) we now have a relation between $G\left(-\varepsilon_{e}, 1,-\varepsilon_{e}, 1,1\right)$ and $G\left(1-\varepsilon_{e}, 1,-\varepsilon_{e}, 1,1\right)$. Expressing the former as a function of the later, yields, in graphical form:

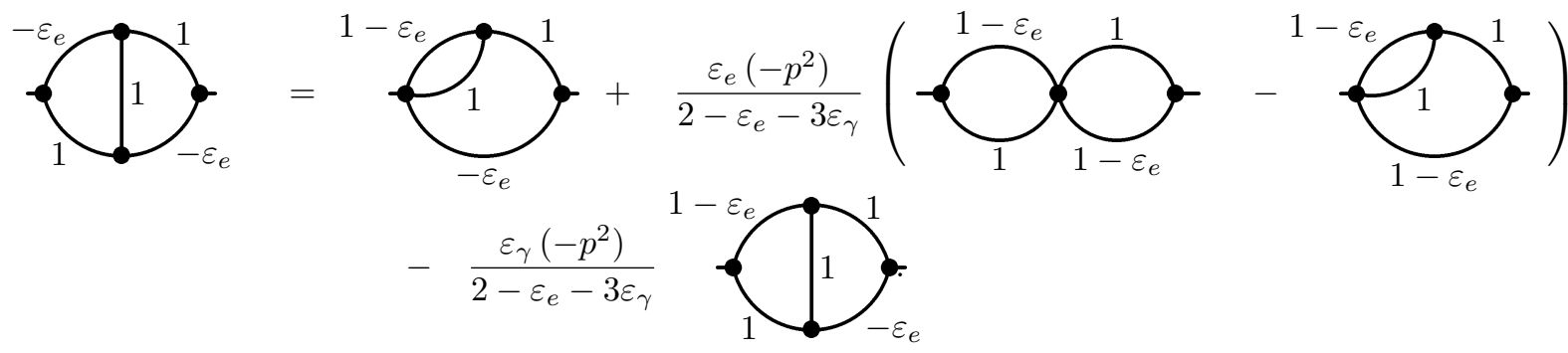

Similarly to the previous cases, the recursively one-loop diagrams appearing in the right-hand side of Eq. (56) can be expressed in terms of the one-loop master integrals entering the expression of the fermion self-energy Eq. (45). Together with Eq. (51), the final result reads:

$$
\begin{aligned}
G\left(-\varepsilon_{e}, 1,-\varepsilon_{e}, 1,1\right) & =-\frac{3 d_{\gamma}-d_{e}-6}{d_{\gamma}+d_{e}-6} G\left(1,1-\varepsilon_{e}\right) G\left(1-\varepsilon_{e}, \varepsilon_{\gamma}\right) \\
& +\frac{1}{2 d_{\gamma}+d_{e}-8}\left(\frac{\left(d_{\gamma}-4\right)\left(d_{\gamma}+d_{e}-6\right)}{2 d_{\gamma}+d_{e}-10}+d_{\gamma}-d_{e}\right) G^{2}\left(1,1-\varepsilon_{e}\right) \\
& +\frac{\left(d_{\gamma}-4\right)^{2}}{\left(2 d_{\gamma}+d_{e}-10\right)\left(2 d_{\gamma}+d_{e}-8\right)} G\left(1-\varepsilon_{e}, 1,1-\varepsilon_{e}, 1,1\right) .
\end{aligned}
$$

Eqs. (51), (54) and (57) constitute the central results of this paragraph. They show that all three complicated diagrams appearing in Eq. (45) are related: they can all be expressed in terms of the ultra-violet convergent diagram $G\left(1-\varepsilon_{e}, 1,1-\varepsilon_{e}, 1,1\right)$ with a factor proportional to $\varepsilon_{\gamma}$.

With the help of Eqs. (51), (54) and (57) the two-loop self-energy associated with the crossed photon diagram, 
Eq. 45, reads:

$$
\begin{aligned}
& \Sigma_{V 2 c}\left(p^{2}\right)=-\frac{e^{4} \Gamma^{2}\left(1-\varepsilon_{e}\right)}{(4 \pi)^{d_{\gamma}}\left(-p^{2}\right)^{2 \varepsilon_{\gamma}}} \frac{d_{e}-2}{2} \times\left\{\left[d_{e}-4-2 \xi \frac{\left(d_{e}-2\right)^{2}}{d_{\gamma}+d_{e}-4}+\xi^{2} \frac{d_{e}-2}{2}\right.\right. \\
& \left.-\frac{\left(d_{\gamma}+d_{e}-6\right)\left(d_{\gamma}\left(d_{e}-4\right)+8\right)}{\left(2 d_{\gamma}+d_{e}-10\right)\left(2 d_{\gamma}+d_{e}-8\right)}-\frac{4\left(d_{\gamma}-d_{e}\right)}{d_{\gamma}+d_{e}-4}-\frac{d_{\gamma}-d_{e}}{2 d_{\gamma}+d_{e}-8}\left(d_{e}-8-4 \frac{d_{\gamma}+d_{e}-6}{d_{\gamma}+d_{e}-4}\right)\right] G^{2}\left(1,1-\varepsilon_{e}\right) \\
& +\left[2 d_{e}-4+8 \frac{d_{e}-2}{d_{\gamma}+d_{e}-4}+\frac{8\left(d_{\gamma}-1\right)}{d_{\gamma}-4}+4 \xi \frac{\left(d_{e}-2\right)\left(d_{\gamma}-3\right)}{d_{\gamma}+d_{e}-4}-\xi^{2}\left(d_{\gamma}-3\right)\right. \\
& \left.+\frac{2\left(d_{e}-8\right)\left(d_{\gamma}-d_{e}\right)}{d_{\gamma}+d_{e}-6}-\frac{4\left(d_{\gamma}-2\right)\left(d_{\gamma}-d_{e}\right)}{\left(d_{\gamma}-4\right)\left(d_{\gamma}+d_{e}-4\right)}\right] G\left(1,1-\varepsilon_{e}\right) G\left(1-\varepsilon_{e}, \varepsilon_{\gamma}\right) \\
& \left.-\frac{\left(d_{\gamma}-4\right)\left(d_{\gamma}\left(d_{e}-4\right)+8\right)}{\left(2 d_{\gamma}+d_{e}-8\right)\left(2 d_{\gamma}+d_{e}-10\right)} G\left(1-\varepsilon_{e}, 1,1-\varepsilon_{e}, 1,1\right)\right\} \text {. }
\end{aligned}
$$

\section{Total two-loop fermion self-energy (explicit expression)}

The sum of all the self-energy contributions, Eqs. (41), (43) and Eq. (58), yields the total two-loop fermion selfenergy in an arbitrary gauge:

$$
\begin{aligned}
& \Sigma_{V 2}\left(p^{2}\right)=\frac{e^{4} \Gamma^{2}\left(1-\varepsilon_{e}\right)}{(4 \pi)^{d_{\gamma}}\left(-p^{2}\right)^{2 \varepsilon_{\gamma}}} \frac{d_{e}-2}{2}\left\{d \frac{d_{e}-2}{2 d_{\gamma}-d_{e}-6} G(1,1) G\left(1, \varepsilon_{\gamma}-\varepsilon_{e}\right)\right. \\
& +\left[4-d_{e}+2 \xi \frac{\left(d_{e}-2\right)^{2}}{d_{\gamma}+d_{e}-4}-\xi^{2} \frac{d_{e}-2}{2}+\frac{\left(d_{\gamma}+d_{e}-6\right)\left(d_{\gamma}\left(d_{e}-4\right)+8\right)}{\left(2 d_{\gamma}+d_{e}-10\right)\left(2 d_{\gamma}+d_{e}-8\right)}\right. \\
& \left.+\frac{4\left(d_{\gamma}-d_{e}\right)}{d_{\gamma}+d_{e}-4}+\frac{d_{\gamma}-d_{e}}{2 d_{\gamma}+d_{e}-8}\left(d_{e}-8-4 \frac{d_{\gamma}+d_{e}-6}{d_{\gamma}+d_{e}-4}\right)\right] G^{2}\left(1,1-\varepsilon_{e}\right) \\
& +\left[4-2 d_{e}-\frac{8\left(d_{e}-2\right)}{d_{\gamma}+d_{e}-4}-\frac{8\left(d_{\gamma}-1\right)}{d_{\gamma}-4}-4 \xi \frac{\left(d_{e}-2\right)\left(d_{\gamma}-3\right)}{d_{\gamma}+d_{e}-4}+\xi^{2}\left(d_{\gamma}-3\right)\right. \\
& +\frac{\left(d_{\gamma}-3\right)\left(d_{\gamma}+d_{e}-4\right)}{d_{\gamma}-4}\left(\frac{2\left(d_{e}-2\right)}{d_{\gamma}+d_{e}-4}-\xi\right)^{2} \\
& \left.-\frac{2\left(d_{e}-8\right)\left(d_{\gamma}-d_{e}\right)}{d_{\gamma}+d_{e}-6}+\frac{4\left(d_{\gamma}-2\right)\left(d_{\gamma}-d_{e}\right)}{\left(d_{\gamma}-4\right)\left(d_{\gamma}+d_{e}-4\right)}\right] G\left(1,1-\varepsilon_{e}\right) G\left(1-\varepsilon_{e}, \varepsilon_{\gamma}\right)+ \\
& \left.+\frac{\left(d_{\gamma}-4\right)\left(d_{\gamma}\left(d_{e}-4\right)+8\right)}{\left(2 d_{\gamma}+d_{e}-8\right)\left(2 d_{\gamma}+d_{e}-10\right)} G\left(1-\varepsilon_{e}, 1,1-\varepsilon_{e}, 1,1\right)\right\} .
\end{aligned}
$$

As explained in the previous paragraph, the last line of Eq. 59 contains the UV-convergent diagram $G\left(1-\varepsilon_{e}, 1,1-\right.$ $\left.\varepsilon_{e}, 1,1\right)$ with a prefactor proportional to $d_{\gamma}-4$. It therefore vanishes for all $\mathrm{RQED}_{4, d_{e}}$ and in particular in $\mathrm{RQED}_{4,3}$ which enables us to avoid computing the complicated diagram $G(1 / 2,1,1 / 2,1,1)$ and obtain an expansion valid to $\mathrm{O}\left(\varepsilon_{\gamma}\right)$ for the two-loop fermion self-energy.

Eq. (59) simplifies for usual QEDs where $d_{\gamma}=d_{e}$ corresponding to $\varepsilon_{e}=0$. In this case, the complicated diagram reduces to the well known $G(1,1,1,1,1)$ which is given by Eq. (36) and Eq. (59) becomes:

$$
\begin{aligned}
& \Sigma_{V 2}\left(p^{2}\right)=\frac{e^{4}\left(-p^{2}\right)^{-2 \varepsilon_{\gamma}}}{(4 \pi)^{d_{\gamma}}}\left(d_{\gamma}-2\right)\left\{2 N_{F} \frac{d_{\gamma}-2}{d_{\gamma}-6} G(1,1) G\left(1, \varepsilon_{\gamma}\right)-\frac{1}{4}\left(d_{\gamma}-6+\left(d_{\gamma}-2\right) a^{2}\right) G^{2}(1,1)\right. \\
& \left.+\frac{1}{2} \frac{d_{\gamma}-3}{d_{\gamma}-4}\left(a^{2}\left(3 d_{\gamma}-8\right)-d_{\gamma}-4\right) G(1,1) G\left(1, \varepsilon_{\gamma}\right)\right\},
\end{aligned}
$$

where Eq. (31) has been used. Eq. (60) agrees with well known results in the literature, see e.g., Ref. 11]. 


\section{ANOMALOUS DIMENSION OF THE FERMION FIELD AND RENORMALIZED FERMION PROPAGATOR UP TO TWO LOOPS}

We now compute the renormalization constant associated to the fermion field $Z_{\psi}$, the related anomalous dimension $\gamma_{\psi}$ and renormalized propagator $S_{r}(p ; \mu)$ up to two loops. To achieve this task we use for RQED well known methods used for usual QEDs, see, e.g., Ref. [11].

\section{A. Renormalization}

In the following, we shall expand the one-loop and two-loop fermion self-energies, for fixed $\varepsilon_{e}$, in Laurent series in $\varepsilon_{\gamma}$. For this purpose, we introduce two functions, $\sigma_{1}$ and $\sigma_{2}$, which are defined with the help of the expressions of the one-loop (Eq. (34b)) and two-loop (Eq. (59)) fermion self-energies as follows:

$$
\begin{aligned}
& \Sigma_{V 1}\left(p^{2}\right)=\frac{e^{2}\left(-p^{2}\right)^{-\varepsilon_{\gamma}}}{(4 \pi)^{d_{\gamma} / 2}} \sigma_{1}\left(\varepsilon_{e}, \varepsilon_{\gamma}, a\right), \\
& \Sigma_{V 2}\left(p^{2}\right)=\frac{e^{4}\left(-p^{2}\right)^{-2 \varepsilon_{\gamma}}}{(4 \pi)^{d_{\gamma}}} \sigma_{2}\left(\varepsilon_{e}, \varepsilon_{\gamma}, a\right) .
\end{aligned}
$$

As will be seen shortly, it is important to single out in the function $\sigma_{1}$ the part which depends on the (bare) gauge fixing parameter $a$. From Eq. (34b), this yields

$$
\begin{aligned}
& \sigma_{1}\left(\varepsilon_{e}, \varepsilon_{\gamma}, a\right)=\sigma_{1}^{\prime}\left(\varepsilon_{e}, \varepsilon_{\gamma}\right)+a \sigma_{1}^{\prime \prime}\left(\varepsilon_{e}, \varepsilon_{\gamma}\right), \\
& \sigma_{1}^{\prime}\left(\varepsilon_{e}, \varepsilon_{\gamma}\right)=\Gamma\left(1-\varepsilon_{e}\right) \frac{\varepsilon_{e}\left(1-\varepsilon_{e}-\varepsilon_{\gamma}\right)}{2-\varepsilon_{e}-2 \varepsilon_{\gamma}} G\left(1,1-\varepsilon_{e}\right), \\
& \sigma_{1}^{\prime \prime}\left(\varepsilon_{e}, \varepsilon_{\gamma}\right)=-\Gamma\left(1-\varepsilon_{e}\right)\left(1-\varepsilon_{e}-\varepsilon_{\gamma}\right) G\left(1,1-\varepsilon_{e}\right) .
\end{aligned}
$$

On the other hand, in the 2-loop function $\sigma_{2}$ we may single out the three contributions corresponding to the three diagrams of Fig. 4 whose expressions are given by Eqs. (41), 443) and (58). This yields:

$$
\begin{aligned}
& \sigma_{2}\left(\varepsilon_{e}, \varepsilon_{\gamma}, a\right)=\sigma_{a}^{(2)}\left(\varepsilon_{e}, \varepsilon_{\gamma}\right)+\sigma_{b}^{(2)}\left(\varepsilon_{e}, \varepsilon_{\gamma}, a\right)+\sigma_{c}^{(2)}\left(\varepsilon_{e}, \varepsilon_{\gamma}, a\right) \\
& \sigma_{a}^{(2)}\left(\varepsilon_{e}, \varepsilon_{\gamma}\right)=-4 N_{F} \Gamma^{2}\left(1-\varepsilon_{e}\right) \frac{\left(1-\varepsilon_{e}-\varepsilon_{\gamma}\right)^{2}}{1-\varepsilon_{e}+\varepsilon_{\gamma}} G(1,1) G\left(1, \varepsilon_{\gamma}-\varepsilon_{e}\right), \\
& \sigma_{b}^{(2)}\left(\varepsilon_{e}, \varepsilon_{\gamma}, a\right)=-\Gamma^{2}\left(1-\varepsilon_{e}\right) \frac{\left(1-2 \varepsilon_{\gamma}\right)\left(1-\varepsilon_{e}-\varepsilon_{\gamma}\right)\left(\varepsilon_{e}-a\left(2-\varepsilon_{e}-2 \varepsilon_{\gamma}\right)\right)^{2}}{\varepsilon_{\gamma}\left(2-\varepsilon_{e}-2 \varepsilon_{\gamma}\right)} G\left(1,1-\varepsilon_{e}\right) G\left(1-\varepsilon_{e}, \varepsilon_{\gamma}\right), \\
& \sigma_{c}^{(2)}\left(\varepsilon_{e}, \varepsilon_{\gamma}, a\right)=\Gamma^{2}\left(1-\varepsilon_{e}\right)\left(1-\varepsilon_{e}-\varepsilon_{\gamma}\right) \times \\
& \times\left\{G ^ { 2 } ( 1 , 1 - \varepsilon _ { e } ) \left[2\left(\varepsilon_{e}+\varepsilon_{\gamma}\right)+4(1-a) \frac{\left(1-\varepsilon_{e}-\varepsilon_{\gamma}\right)^{2}}{2-\varepsilon_{e}-2 \varepsilon_{\gamma}}-(1-a)^{2}\left(1-\varepsilon_{e}-\varepsilon_{\gamma}\right)\right.\right. \\
& \left.+2 \frac{\left(1-\varepsilon_{e}-2 \varepsilon_{\gamma}\right)\left[2-\left(2-\varepsilon_{\gamma}\right)\left(\varepsilon_{e}+\varepsilon_{\gamma}\right)\right]}{\left(1-\varepsilon_{e}-3 \varepsilon_{\gamma}\right)\left(2-\varepsilon_{e}-3 \varepsilon_{\gamma}\right)}+\frac{4 \varepsilon_{e}}{2-\varepsilon_{e}-2 \varepsilon_{\gamma}}-\frac{2 \varepsilon_{e}}{2-\varepsilon_{e}-3 \varepsilon_{\gamma}}\left(2+\varepsilon_{e}+\varepsilon_{\gamma}+2 \frac{1-\varepsilon_{e}-2 \varepsilon_{\gamma}}{2-\varepsilon_{e}-2 \varepsilon_{\gamma}}\right)\right] \\
& -G\left(1,1-\varepsilon_{e}\right) G\left(1-\varepsilon_{e}, \varepsilon_{\gamma}\right)\left[4-4 \varepsilon_{e}-4 \varepsilon_{\gamma}+\frac{8\left(1-\varepsilon_{e}-\varepsilon_{\gamma}\right)}{2-\varepsilon_{e}-2 \varepsilon_{\gamma}}-\frac{4\left(3-2 \varepsilon_{\gamma}\right)}{\varepsilon_{\gamma}}+4(1-a) \frac{\left(1-\varepsilon_{e}-\varepsilon_{\gamma}\right)\left(1-2 \varepsilon_{\gamma}\right)}{2-\varepsilon_{e}-2 \varepsilon_{\gamma}}\right. \\
& \left.-(1-a)^{2}\left(1-2 \varepsilon_{\gamma}\right)-4 \frac{\varepsilon_{e}\left(2+\varepsilon_{e}+\varepsilon_{\gamma}\right)}{1-\varepsilon_{e}-2 \varepsilon_{\gamma}}+4 \frac{\varepsilon_{e}\left(1-\varepsilon_{\gamma}\right)}{\varepsilon_{\gamma}\left(2-\varepsilon_{e}-2 \varepsilon_{\gamma}\right)}\right] \\
& \left.-\frac{2 \varepsilon_{\gamma}\left[2-\left(2-\varepsilon_{\gamma}\right)\left(\varepsilon_{e}+\varepsilon_{\gamma}\right)\right]}{\left(2-\varepsilon_{e}-3 \varepsilon_{\gamma}\right)\left(1-\varepsilon_{e}-3 \varepsilon_{\gamma}\right)} G\left(1-\varepsilon_{e}, 1,1-\varepsilon_{e}, 1,1\right)\right\} .
\end{aligned}
$$

These equations are no more than a re-writing of the two-loop self-energies, Eqs. (41), (43) and (58), in terms of the $\varepsilon$-parameters instead of the dimensions.

In order to implement the renormalization procedure all bare quantities (charge and gauge fixing parameter) appearing in the self-energies Eqs. (61) have to be expressed in terms of renormalized ones:

$$
\frac{e^{2}}{(4 \pi)^{d_{\gamma} / 2}}=Z_{\alpha}(\alpha(\mu)) \frac{\alpha(\mu)}{4 \pi} \mu^{2 \varepsilon_{\gamma}} e^{\gamma_{E} \varepsilon_{\gamma}}, \quad a=Z_{A}(\alpha(\mu)) a_{r}(\mu),
$$


where Eqs. (16) and 15) were used. For an expansion to $\mathrm{O}\left(\alpha^{2}\right)$ it is enough to know the coupling and gauge field renormalizations at one-loop. From Ref. [20] they read:

$$
Z_{\alpha}(\alpha)=Z_{A}^{-1}(\alpha)=1+z_{\alpha} \frac{\alpha}{4 \pi \varepsilon_{\gamma}}+\mathrm{O}\left(\alpha^{2}\right), \quad \gamma_{A}(\alpha)=2 z_{\alpha} \frac{\alpha}{4 \pi}, \quad \beta(\alpha)=-2 \varepsilon_{\gamma}+2 z_{\alpha} \frac{\alpha}{4 \pi},
$$

where the coefficient $z_{\alpha}$ depends of the theory under consideration:

$$
z_{\alpha}=\frac{4 N_{F}}{3}\left(\mathrm{QED}_{4}\right), \quad z_{\alpha}=0\left(\mathrm{RQED}_{4,3}\right)
$$

Summing the one-loop and two-loop contributions, the total self-energy up to two loops can then be written as:

$$
\Sigma_{V}\left(p^{2}\right)=\frac{\alpha}{4 \pi} e^{\left(\gamma_{E}-L_{p}\right) \varepsilon_{\gamma}} \sigma_{1}\left(a_{r}\right)+\left(\frac{\alpha}{4 \pi}\right)^{2}\left(\frac{z_{\alpha}}{\varepsilon_{\gamma}} e^{\left(\gamma_{E}-L_{p}\right) \varepsilon_{\gamma}} \sigma_{1}^{\prime}+e^{2\left(\gamma_{E}-L_{p}\right) \varepsilon_{\gamma}} \sigma_{2}\left(a_{r}\right)\right)+\mathrm{O}\left(\alpha^{3}\right),
$$

where $L_{p}=\log \left(-p^{2} / \mu^{2}\right)$ and the functions $\sigma_{1}=\sigma_{1}^{\prime}+a_{r} \sigma_{1}^{\prime \prime}$ and $\sigma_{2}$ now depend on the renormalized gauge fixing parameter. With the help of Eq. (67) the expansion of the fermion propagator Eq. (14) reads:

$$
-i \not p S(p)=1+\frac{\alpha}{4 \pi} e^{\left(\gamma_{E}-L_{p}\right) \varepsilon_{\gamma}} \sigma_{1}\left(a_{r}\right)+\left(\frac{\alpha}{4 \pi}\right)^{2}\left[\frac{z_{\alpha}}{\varepsilon_{\gamma}} e^{\left(\gamma_{E}-L_{p}\right) \varepsilon_{\gamma}} \sigma_{1}^{\prime}+e^{2\left(\gamma_{E}-L_{p}\right) \varepsilon_{\gamma}}\left(\sigma_{1}^{2}\left(a_{r}\right)+\sigma_{2}\left(a_{r}\right)\right)\right]+\mathrm{O}\left(\alpha^{3}\right) .
$$

The Laurent series associated with the various functions entering Eq. 68 can be written as:

$$
\begin{aligned}
& e^{\left(\gamma_{E}-L_{p}\right) \varepsilon_{\gamma}} \sigma_{1}^{\prime}=\frac{c_{10}^{\prime}}{\varepsilon_{\gamma}}+c_{11}^{\prime}+c_{12}^{\prime} \varepsilon_{\gamma}+\cdots, \quad e^{\left(\gamma_{E}-L_{p}\right) \varepsilon_{\gamma}} \sigma_{1}^{\prime \prime}=\frac{c_{10}^{\prime \prime}}{\varepsilon_{\gamma}}+c_{11}^{\prime \prime}+c_{12}^{\prime \prime} \varepsilon_{\gamma}+\cdots, \\
& e^{2\left(\gamma_{E}-L_{p}\right) \varepsilon_{\gamma}}\left(\sigma_{1}^{2}+\sigma_{2}\right)=\frac{c_{20}}{\varepsilon_{\gamma}^{2}}+\frac{c_{21}}{\varepsilon_{\gamma}}+c_{22}+\cdots,
\end{aligned}
$$

where the dots indicate higher order terms in $\varepsilon_{\gamma}$. In Eq. $69 \mathrm{~b}$ the two-loop contribution has been expanded up to $\mathrm{O}(1)$ in $\varepsilon_{\gamma}$ which is all we need for two-loop renormalization and is within the accuracy of our loop calculations in $\mathrm{RQED}_{4, d_{e}}$. With these notations Eq. (68) can then be re-written as:

$$
-i \not p S(p)=1+\frac{\alpha}{4 \pi}\left(\frac{c_{10}}{\varepsilon_{\gamma}}+c_{11}+c_{12} \varepsilon_{\gamma}+\cdots\right)+\left(\frac{\alpha}{4 \pi}\right)^{2}\left(\frac{c_{20}+z_{\alpha} c_{10}^{\prime}}{\varepsilon_{\gamma}^{2}}+\frac{c_{21}+z_{\alpha} c_{11}^{\prime}}{\varepsilon_{\gamma}}+c_{22}+z_{\alpha} c_{12}^{\prime}+\cdots\right)+\mathrm{O}\left(\alpha^{3}\right)(, 70)
$$

where the one-loop coefficients $c_{1 j}=c_{1 j}^{\prime}+a_{r} c_{1 j}^{\prime \prime}$ are defined by Eqs. 69a and the coefficients $c_{2 j}$, which also depend on $a_{r}$, are defined by Eq. 69b).

In the $\overline{\mathrm{MS}}$ scheme all singular terms are factorized in the renormalization constant $Z_{\psi}$ while the regular terms contribute to the renormalized propagator $S_{r}$, yielding general expressions of the form:

$$
\begin{aligned}
Z_{\psi}\left(\alpha, a_{r}\right) & =1+\frac{\alpha}{4 \pi} \frac{z_{1}}{\varepsilon_{\gamma}}+\left(\frac{\alpha}{4 \pi}\right)^{2}\left(\frac{z_{20}}{\varepsilon_{\gamma}^{2}}+\frac{z_{21}}{\varepsilon_{\gamma}}\right)+\mathrm{O}\left(\alpha^{3}\right), \\
-i \not p S_{r}(p ; \mu) & =1+\frac{\alpha}{4 \pi}\left(r_{1}+r_{11} \varepsilon_{\gamma}+\cdots\right)+\left(\frac{\alpha}{4 \pi}\right)^{2}\left(r_{2}+\cdots\right)+\mathrm{O}\left(\alpha^{3}\right),
\end{aligned}
$$

where the coefficients $z_{i j}$ and $r_{i j}$ are to be determined. This can be done with the help of Eq. (17) by identifying, at a given loop order, the coefficients of equal powers of $\varepsilon_{\gamma}$. The result reads:

$$
\begin{aligned}
& z_{1}=c_{10}, \quad z_{20}=c_{20}+z_{\alpha} c_{10}^{\prime}, \quad z_{21}=c_{21}+z_{\alpha} c_{11}^{\prime}-c_{10} c_{11}, \\
& r_{1}=c_{11}, \quad r_{11}=c_{12}, \quad r_{2}=c_{22}+z_{\alpha} c_{12}^{\prime}-c_{10} c_{12} \text {. }
\end{aligned}
$$

We are now in a position to present a general expression for the anomalous scaling dimension of the fermion field. In partial derivative form the latter reads

$$
\gamma_{\psi}\left(\alpha(\mu), a_{r}(\mu)\right)=\frac{d \log Z_{\psi}\left(\alpha(\mu), a_{r}(\mu)\right)}{d \log \mu}=\beta(\alpha(\mu)) \frac{\partial \log Z_{\psi}\left(\alpha, a_{r}\right)}{\partial \log \alpha}-\gamma_{A}(\alpha) \frac{\partial \log Z_{\psi}\left(\alpha, a_{r}\right)}{\partial \log a_{r}},
$$

where the one-loop beta-function and anomalous scaling dimension of the gauge field are given by Eqs. 65). From Eqs. (71a), 72a and (65), we recover the fact, well known in usual QED, that the simple poles of $Z_{\psi}$ determine the anomalous scaling dimension:

$$
\gamma_{\psi}\left(\alpha, a_{r}\right)=-2 z_{1} \frac{\alpha}{4 \pi}-4 z_{21}\left(\frac{\alpha}{4 \pi}\right)^{2}+\mathrm{O}\left(\alpha^{3}\right)
$$


and that, together with the beta function, they also determine the higher order poles:

$$
z_{20}=\frac{z_{1}^{2}+z_{\alpha} c_{10}^{\prime}}{2}
$$

The constraint Eq. 75 comes from the fact that the anomalous dimension has to be finite as $\varepsilon_{\gamma} \rightarrow 0$.

\section{B. Case of $\operatorname{RQED}_{4, d_{e}}$}

In this paragraph we shall compute the anomalous dimension of the fermion field and renormalized fermion propagator in a general theory of $\mathrm{RQED}_{4, d_{e}}$. In order to do so, we expand the $\sigma$ functions of Eqs. 62 and 63 in Laurent series in $\varepsilon_{\gamma}$ for arbitrary $\varepsilon_{e}$. The coefficients of the expansions read:

$$
\begin{aligned}
& e^{\left(\gamma_{E}-L_{p}\right) \varepsilon_{\gamma}} \sigma_{1}^{\prime}=\frac{\varepsilon_{e}}{\left(2-\varepsilon_{e}\right) \varepsilon_{\gamma}}-\frac{\varepsilon_{e}}{2-\varepsilon_{e}}\left(\bar{L}_{p}-\frac{2}{2-\varepsilon_{e}}\right) \\
& \quad+\frac{\varepsilon_{e}}{2\left(2-\varepsilon_{e}\right)}\left(\bar{L}_{p}\left(\bar{L}_{p}-\frac{4}{2-\varepsilon_{e}}\right)+2 \zeta_{2}-3 \Psi_{2}\left(2-\varepsilon_{e}\right)+\frac{8}{\left(2-\varepsilon_{e}\right)^{2}}\right) \varepsilon_{\gamma}+\mathrm{O}\left(\varepsilon_{\gamma}^{2}\right) \\
& e^{\left(\gamma_{E}-L_{p}\right) \varepsilon_{\gamma}} \sigma_{1}^{\prime \prime}=-\frac{1}{\varepsilon_{\gamma}}+\bar{L}_{p}-\frac{1}{2}\left(\bar{L}_{p}^{2}+2 \zeta_{2}-3 \Psi_{2}\left(2-\varepsilon_{e}\right)\right) \varepsilon_{\gamma}+\mathrm{O}\left(\varepsilon_{\gamma}^{2}\right) \\
& e^{2\left(\gamma_{E}-L_{p}\right) \varepsilon_{\gamma}}\left(\sigma_{1}^{2}+\sigma_{2}\right)=\frac{1}{2}\left(a_{r}-\frac{\varepsilon_{e}}{2-\varepsilon_{e}}\right)^{2} \frac{1}{\varepsilon_{\gamma}^{2}}-\left(\left(a_{r}-\frac{\varepsilon_{e}}{2-\varepsilon_{e}}\right)^{2} \bar{L}_{p}+2 a_{r} \frac{\varepsilon_{e}}{\left(2-\varepsilon_{e}\right)^{2}}\right. \\
& \left.\quad-2 \frac{\varepsilon_{e}^{2}\left(10-3 \varepsilon_{e}\right)-11 \varepsilon_{e}+3}{\left(1-\varepsilon_{e}\right)\left(2-\varepsilon_{e}\right)^{3}}+2 N_{F} K_{1} \frac{\varepsilon_{e}}{2-\varepsilon_{e}}\right) \frac{1}{\varepsilon_{\gamma}} \\
& \quad+\left(a_{r}-\frac{\varepsilon_{e}}{2-\varepsilon_{e}}\right)^{2}\left(\bar{L}_{p}^{2}+\frac{3}{2} \zeta_{2}-2 \Psi_{2}\left(2-\varepsilon_{e}\right)\right)+4 a_{r} \frac{\varepsilon_{e}}{\left(2-\varepsilon_{e}\right)^{2}}\left(\bar{L}_{p}-\frac{1}{2-\varepsilon_{e}}\right) \\
& \quad+4 \frac{3-2 \varepsilon_{e}}{\left(2-\varepsilon_{e}\right)^{2}}\left(\Psi_{2}\left(1-\varepsilon_{e}\right)-\zeta_{2}\right)-4 \bar{L}_{p} \frac{\varepsilon_{e}^{2}\left(10-3 \varepsilon_{e}\right)-11 \varepsilon_{e}+3}{\left(1-\varepsilon_{e}\right)\left(2-\varepsilon_{e}\right)^{3}}+2 \frac{\varepsilon_{e}^{2}\left(32-10 \varepsilon_{e}\right)-23 \varepsilon_{e}-7}{\left(1-\varepsilon_{e}\right)\left(2-\varepsilon_{e}\right)^{4}} \\
& \quad-2 \frac{\varepsilon_{e}^{2}}{\left(1-\varepsilon_{e}\right)^{2}\left(2-\varepsilon_{e}\right)^{2}}+4 N_{F} K_{1} \frac{\varepsilon_{e}}{2-\varepsilon_{e}}\left(\bar{L}_{p}+\frac{1}{2}\left(\bar{\Psi}_{1}-\bar{\Psi}_{2}\right)+\frac{1}{\varepsilon_{e}\left(1-\varepsilon_{e}\right)\left(2-\varepsilon_{e}\right)}-\frac{1}{2-\varepsilon_{e}}\right)+\mathrm{O}\left(\varepsilon_{\gamma}\right)
\end{aligned}
$$

where

$$
\begin{aligned}
& \bar{L}_{p}=L_{p}-\Psi_{1}\left(2-\varepsilon_{e}\right)+\Psi_{1}(1), \quad K_{1}=\frac{\Gamma^{3}\left(1-\varepsilon_{e}\right) \Gamma\left(\varepsilon_{e}\right)}{\Gamma\left(2-2 \varepsilon_{e}\right)}, \\
& \bar{\Psi}_{1}=\Psi_{1}\left(1-\varepsilon_{e}\right)-\Psi_{1}(1), \quad \bar{\Psi}_{2}=\Psi_{1}\left(\varepsilon_{e}\right)-2 \Psi_{1}\left(1-\varepsilon_{e}\right)+2 \Psi_{1}\left(2-2 \varepsilon_{e}\right)-\Psi_{1}(1),
\end{aligned}
$$

and $\Psi_{1}$ and $\Psi_{2}$ are the digamma and trigamma functions, respectively. From Eqs. (76) the coefficients $c_{1 j}$ and $c_{2 j}$ can be deduced.

Focusing for the moment on the singular contributions, which are obtained by combining Eqs. 76] with Eqs. 72a, yields:

$$
\begin{aligned}
& z_{1}=-\left(a_{r}-\frac{\varepsilon_{e}}{2-\varepsilon_{e}}\right), \quad z_{20}=\frac{1}{2}\left(a_{r}-\frac{\varepsilon_{e}}{2-\varepsilon_{e}}\right)^{2}+z_{\alpha} \frac{\varepsilon_{e}}{2-\varepsilon_{e}}, \\
& z_{21}=-2 N_{F} K_{1} \frac{\varepsilon_{e}}{2-\varepsilon_{e}}+2 \frac{\left(3-2 \varepsilon_{e}\right)\left[1-\varepsilon_{e}\left(3-\varepsilon_{e}\right)\right]}{\left(1-\varepsilon_{e}\right)\left(2-\varepsilon_{e}\right)^{3}}-z_{\alpha} \frac{\varepsilon_{e}}{2-\varepsilon_{e}}\left(\bar{L}_{p}-\frac{2}{2-\varepsilon_{e}}\right),
\end{aligned}
$$

where all gauge depend terms cancel out from $z_{21}$. In order for the consistency relation, Eq. $(75)$, to be valid we see from Eqs. $78 \mathrm{a}$ ) that we must either have $\varepsilon_{e}=0$ if $z_{\alpha} \neq 0$ which is the case of $\mathrm{QED}_{4}$ or $z_{\alpha}=0$ if $\varepsilon_{e} \neq 0$ which is the case of $\overline{\mathrm{RQED}}_{4,3}$, see Eq. (66). Taking this constraint into account we see that momentum dependent terms also cancel out from $z_{21}$. Then, the general expression of the anomalous dimension of the fermion field in $\operatorname{RQED}_{4, d_{e}}$ reads

$$
\gamma_{\psi}\left(\alpha, a_{r}\right)=2\left(a_{r}-\frac{\varepsilon_{e}}{2-\varepsilon_{e}}\right) \frac{\alpha}{4 \pi}+8\left(N_{F} K_{1} \frac{\varepsilon_{e}}{2-\varepsilon_{e}}-\frac{\left(3-2 \varepsilon_{e}\right)\left[1-\varepsilon_{e}\left(3-\varepsilon_{e}\right)\right]}{\left(1-\varepsilon_{e}\right)\left(2-\varepsilon_{e}\right)^{3}}\right)\left(\frac{\alpha}{4 \pi}\right)^{2}+\mathrm{O}\left(\alpha^{3}\right) .
$$


It is important that Eq. 790 does not depend on the external momenta and digamma functions. This property is an extension of the rule ${ }^{35}$ for the results of the anomalous dimensions in standard QFT $\left(\varepsilon_{e}=0\right.$ and $\left.\varepsilon_{\gamma} \rightarrow 0\right)$, where the corresponding anomalous dimensions do not depend on the external momenta and (in $\overline{M S}$-scheme) on $\gamma_{E}$ and $\zeta_{2}$. Note that in the framework of $\mathrm{RQED}_{4,3}$ there is a contribution of $\zeta_{2}$ in the corresponding anomalous dimension (see Eq. (84) below) but this contribution appears, not from the expansion of $\Gamma$-functions in $\varepsilon_{\gamma}$ but from the factor $K_{1}$ in the expression $G(1,1)$, see App. A

Finally, the finite part can be obtained in a similar way by combining Eqs. (76) with Eqs. (72b). This yields:

$$
\begin{aligned}
r_{1} & =\left(a_{r}-\frac{\varepsilon_{e}}{2-\varepsilon_{e}}\right) \bar{L}_{p}+\frac{2 \varepsilon_{e}}{\left(2-\varepsilon_{e}\right)^{2}}, \\
r_{11} & =-\frac{1}{2}\left(a_{r}-\frac{\varepsilon_{e}}{2-\varepsilon_{e}}\right)\left(\bar{L}_{p}^{2}+2 \zeta_{2}-3 \Psi_{2}\left(2-\varepsilon_{e}\right)\right)-\frac{2 \varepsilon_{e}}{\left(2-\varepsilon_{e}\right)^{2}}\left(\bar{L}_{p}-\frac{2}{2-\varepsilon_{e}}\right), \\
r_{2} & =\frac{1}{2}\left(a_{r}-\frac{\varepsilon_{e}}{2-\varepsilon_{e}}\right)^{2}\left(\bar{L}_{p}^{2}+\zeta_{2}-\Psi_{2}\left(2-\varepsilon_{e}\right)\right)+2 a_{r} \frac{\varepsilon_{e}}{\left(2-\varepsilon_{e}\right)^{2}} \bar{L}_{p}+4 \frac{3-2 \varepsilon_{e}}{\left(2-\varepsilon_{e}\right)^{2}}\left(\Psi_{2}\left(1-\varepsilon_{e}\right)-\zeta_{2}\right) \\
& -2 \bar{L}_{p} \frac{6-22 \varepsilon_{e}+19 \varepsilon_{e}^{2}-5 \varepsilon_{e}^{3}}{\left(1-\varepsilon_{e}\right)\left(2-\varepsilon_{e}\right)^{3}}-2 \frac{7+23 \varepsilon_{e}-30 \varepsilon_{e}^{2}+8 \varepsilon_{e}^{3}}{\left(1-\varepsilon_{e}\right)\left(2-\varepsilon_{e}\right)^{4}}-2 \frac{\varepsilon_{e}^{2}}{\left(1-\varepsilon_{e}\right)^{2}\left(2-\varepsilon_{e}\right)^{2}} \\
& +4 N_{F} K_{1} \frac{\varepsilon_{e}}{2-\varepsilon_{e}}\left(\bar{L}_{p}+\frac{1}{2}\left(\bar{\Psi}_{1}-\bar{\Psi}_{2}\right)+\frac{1}{\varepsilon_{e}\left(1-\varepsilon_{e}\right)\left(2-\varepsilon_{e}\right)}-\frac{1}{2-\varepsilon_{e}}\right) .
\end{aligned}
$$

A few remarks are in order concerning the above results starting from Eqs. (76). The Laurent series in $\varepsilon_{\gamma}$ are plagued by singularities for some values of $\varepsilon_{e}$. The singularity at $\varepsilon_{e}=2$ (case of a 0 -brane or quantum mechanics) reflects the fact that the self-energy of a point-like particle is ill-defined already at one-loop. The singularity at $\varepsilon_{e}=1$ (case of a 1-brane or $\mathrm{RQED}_{4,2}$ ) appears starting from two-loop and is related to the zero-width of the filament, i.e., the higher powers of $\Gamma\left(1-\varepsilon_{e}\right)$, coming from the effective free gauge field propagator (8), as the order increases. Finally, at two-loop, a singularity appears for $\varepsilon_{e}=0$ (case of $\mathrm{QED}_{4}$ ) as can be seen in particular from the last term in Eq. (80c). This singularity is gauge invariant and can be traced back to the UV behaviour of the one-loop polarization operator entering the bubble diagram which is contained in the master integral $G(1,1)$, see App. A The above results are therefore valid only in application to $\mathrm{RQED}_{4,3}$.

\section{Application to massless RQED $_{4,3}$}

In the case of massless $\operatorname{RQED}_{4,3}\left(\varepsilon_{e}=1 / 2\right.$ and $\left.\varepsilon_{\gamma} \rightarrow 0\right)$ the $\varepsilon_{\gamma}$-expansions of Eqs. 76 read:

$$
\begin{aligned}
e^{\left(\gamma_{E}-L_{p}\right) \varepsilon_{\gamma}} \sigma_{1}^{\prime} & =\frac{1}{3 \varepsilon_{\gamma}}+\frac{4-3 \bar{L}_{p}}{9}+\frac{1}{6}\left(\bar{L}_{p}\left(\bar{L}_{p}-\frac{8}{3}\right)-7 \zeta_{2}+\frac{140}{9}\right) \varepsilon_{\gamma}+\mathrm{O}\left(\varepsilon_{\gamma}^{2}\right), \\
e^{\left(\gamma_{E}-L_{p}\right) \varepsilon_{\gamma}} \sigma_{1}^{\prime \prime} & =-\frac{1}{\varepsilon_{\gamma}}+\bar{L}_{p}-\frac{\bar{L}_{p}^{2}-7 \zeta_{2}+12}{2} \varepsilon_{\gamma}+\mathrm{O}\left(\varepsilon_{\gamma}^{2}\right), \\
e^{2\left(\gamma_{E}-L_{p}\right) \varepsilon_{\gamma}}\left(\sigma_{1}^{2}+\sigma_{2}\right) & =\frac{\left(1-3 a_{r}\right)^{2}}{18 \varepsilon_{\gamma}^{2}}-\frac{\left(1-3 a_{r}\right)^{2} \bar{L}_{p}+4 a_{r}+36 \zeta_{2} N_{F}+4}{9 \varepsilon_{\gamma}}+ \\
& +\frac{\left(1-3 a_{r}\right)^{2}}{9}\left(\bar{L}_{p}^{2}-\frac{9}{2} \zeta_{2}+8\right)+\frac{8 a}{9}\left(\bar{L}_{p}-\frac{2}{3}\right)+\frac{64}{9} \zeta_{2}+\frac{8}{9} \bar{L}_{p} \\
& +8 \zeta_{2} N_{F}\left(\bar{L}_{p}+2-\log 4\right)-\frac{824}{81}+\mathrm{O}\left(\varepsilon_{\gamma}\right),
\end{aligned}
$$

where $\bar{L}_{p}=\log \left(-p^{2} / \mu^{2}\right)+\log 4-2$. The coefficients then read:

$$
\begin{aligned}
& z_{1}=\frac{1-3 a_{r}}{3}, \quad z_{20}=\frac{\left(1-3 a_{r}\right)^{2}}{18}, \quad z_{21}=-4 \zeta_{2} N_{F}-\frac{16}{27}, \\
& r_{1}=\frac{4}{9}-\frac{1-3 a_{r}}{3} \bar{L}_{p}, \quad r_{11}=\frac{1-3 a_{r}}{6} \bar{L}_{p}^{2}-\frac{4}{9} \bar{L}_{p}-7 \zeta_{2} \frac{1-3 a_{r}}{6}-6 a_{r}+\frac{70}{27} \\
& r_{2}=\frac{\left(1-3 a_{r}\right)^{2}}{18}\left(\bar{L}_{p}^{2}-2 \zeta_{2}+4\right)+4 \frac{\left(3 a_{r}+7\right) \bar{L}_{p}+48 \zeta_{2}}{27}-8 \zeta_{2} N_{F}\left(\bar{L}_{p}+2-\log 4\right)-\frac{280}{27} .
\end{aligned}
$$


The corresponding renormalization constant and renormalized fermion propagator read:

$$
\begin{aligned}
& Z_{\psi}\left(\alpha, a_{r}\right)=1+\frac{1-3 a_{r}}{3} \frac{\alpha}{4 \pi \varepsilon_{\gamma}}+\left[\frac{\left(1-3 a_{r}\right)^{2}}{18}-4\left(\zeta_{2} N_{F}+\frac{4}{27}\right) \varepsilon_{\gamma}+\cdots\right]\left(\frac{\alpha}{4 \pi \varepsilon_{\gamma}}\right)^{2}+\mathrm{O}\left(\alpha^{3}\right), \\
& -i \not p S_{r}(p ; \mu)=1+\frac{\alpha}{4 \pi}\left[\frac{4}{9}-\frac{1-3 a_{r}}{3} \bar{L}_{p}+\left(\frac{1-3 a_{r}}{6} \bar{L}_{p}^{2}-\frac{4}{9} \bar{L}_{p}-7 \zeta_{2} \frac{1-3 a_{r}}{6}-6 a_{r}+\frac{70}{27}\right) \varepsilon_{\gamma}+\cdots\right] \\
& +\left(\frac{\alpha}{4 \pi}\right)^{2}\left[\frac{\left(1-3 a_{r}\right)^{2}}{18}\left(\bar{L}_{p}^{2}-2 \zeta_{2}+4\right)+4 \frac{\left(3 a_{r}+7\right) \bar{L}_{p}+48 \zeta_{2}}{27}-8 \zeta_{2} N_{F}\left(\bar{L}_{p}+2-\log 4\right)-\frac{280}{27}+\cdots\right]+\mathrm{O}\left(\alpha^{3}\right) .
\end{aligned}
$$

Finally, the anomalous scaling dimension of the fermion field in $\mathrm{RQED}_{4,3}$ reads:

$$
\gamma_{\psi}\left(\alpha, a_{r}\right)=2 \frac{3 a_{r}-1}{3} \frac{\alpha}{4 \pi}+16\left(\zeta_{2} N_{F}+\frac{4}{27}\right)\left(\frac{\alpha}{4 \pi}\right)^{2}+\mathrm{O}\left(\alpha^{3}\right) .
$$

As already noticed in the general formulas, this anomalous dimension does not depend on the external momentum and the two-loop contribution does not depend on the choice of gauge. In order to see how it affects the momentum dependence of the fermion propagator we solve Eq. 26 . For this purpose, we use the fact that: $\beta(\alpha)=\gamma_{A}(\alpha)=0$, as the coupling and gauge field do not renormalize in $\mathrm{RQED}_{4,3}$. The solution then reads:

$$
S_{r}(p ; \mu)=\frac{i}{\not p} s_{r}(1 ; \alpha ; a)\left(\frac{-p^{2}}{\mu^{2}}\right)^{\frac{1}{2} \gamma_{\psi}(\alpha, a)},
$$

where $\gamma_{\psi}$ is given by Eq. (84).

\section{Case of massless $\mathrm{QED}_{4}$}

The case of massless $\mathrm{QED}_{4}$ corresponds to: $\varepsilon_{e}=0$ and $\varepsilon_{\gamma} \rightarrow 0$. Starting from Eq. 60 the $\varepsilon_{\gamma}$-expansions of the self-energies read:

$$
\begin{aligned}
e^{\left(\gamma_{E}-L_{p}\right) \varepsilon_{\gamma}} \sigma_{1}^{\prime} & =0 \\
e^{\left(\gamma_{E}-L_{p}\right) \varepsilon_{\gamma}} \sigma_{1}^{\prime \prime} & =-\frac{1}{\varepsilon_{\gamma}}+L_{p}-1+\frac{\zeta_{2}-L_{p}\left(L_{p}-2\right)-4}{2} \varepsilon_{\gamma}+\mathrm{O}\left(\varepsilon_{\gamma}^{2}\right) \\
e^{2\left(\gamma_{E}-L_{p}\right) \varepsilon_{\gamma}}\left(\sigma_{1}^{2}+\sigma_{2}\right) & =\frac{a_{r}^{2}}{2 \varepsilon_{\gamma}^{2}}+\frac{a_{r}^{2}\left(1-L_{p}\right)+N_{F}+3 / 4}{\varepsilon_{\gamma}}+ \\
& +a_{r}^{2}\left(L_{p}\left(L_{p}-2\right)-\frac{1}{2} \zeta_{2}+3\right)-2 L_{p}\left(N_{F}+\frac{3}{4}\right)+\frac{7}{2} N_{F}+\frac{5}{8}+\mathrm{O}\left(\varepsilon_{\gamma}\right),
\end{aligned}
$$

from which the coefficients $c_{1 j}$ and $c_{2 j}$ can be deduced; in particular, the coefficients $c_{1 j}^{\prime}=0$ in $\mathrm{QED}_{4}$. Substituting these coefficients in Eqs. 72 , yields:

$$
\begin{aligned}
& z_{1}=-a_{r}, \quad z_{20}=\frac{a_{r}^{2}}{2}, \quad z_{21}=N_{F}+\frac{3}{4} \\
& r_{1}=a_{r}\left(L_{p}-1\right), \quad r_{11}=\frac{a_{r}}{2}\left(\zeta_{2}-L_{p}\left(L_{p}-2\right)-4\right) \\
& r_{2}=a_{r}^{2}\left(1+\frac{1}{2} L_{p}\left(L_{p}-2\right)\right)-2 L_{p}\left(N_{F}+\frac{3}{4}\right)+\frac{7}{2} N_{F}+\frac{5}{8} .
\end{aligned}
$$

From Eq. $87 \mathrm{a}$ we see that Eq. 75 is indeed satisfied. Moreover, similarly to the case of QED ${ }_{4,3}$, while $z_{1}$ is gaugevariant, the gauge dependence cancels out in $z_{21}$. Substituting the coefficients of Eqs. (87) in Eqs. (71) yields the following renormalization constant and renormalized fermion propagator:

$$
\begin{aligned}
Z_{\psi}\left(\alpha, a_{r}\right)= & 1-a_{r} \frac{\alpha}{4 \pi \varepsilon_{\gamma}}+\left[\frac{a_{r}^{2}}{2}+\left(N_{F}+\frac{3}{4}\right) \varepsilon_{\gamma}+\cdots\right]\left(\frac{\alpha}{4 \pi \varepsilon_{\gamma}}\right)^{2}+\mathrm{O}\left(\alpha^{3}\right) \\
-i \not p S_{r}(p ; \mu)= & 1+\frac{\alpha}{4 \pi}\left[a_{r}\left(L_{p}-1\right)+\frac{a_{r}}{2}\left(\zeta_{2}-L_{p}\left(L_{P}-2\right)-4\right) \varepsilon_{\gamma}+\cdots\right]+ \\
& +\left(\frac{\alpha}{4 \pi}\right)^{2}\left[a_{r}^{2}\left(1+\frac{1}{2} L_{p}\left(L_{p}-2\right)\right)-2 L_{p}\left(N_{F}+\frac{3}{4}\right)+\frac{7}{2} N_{F}+\frac{5}{8}+\cdots\right]+\mathrm{O}\left(\alpha^{3}\right)
\end{aligned}
$$


Finally, from Eqs. (87a) and (74) the anomalous scaling dimension of the fermion field in $\mathrm{QED}_{4}$ is recovered:

$$
\gamma_{\psi}\left(\alpha, a_{r}\right)=2 a_{r} \frac{\alpha}{4 \pi}-4\left(N_{F}+\frac{3}{4}\right)\left(\frac{\alpha}{4 \pi}\right)^{2}+\mathrm{O}\left(\alpha^{3}\right) .
$$

As in the case of $\mathrm{RQED}_{4,3}$, see Eq. (84), the fermion anomalous dimension of $\mathrm{QED}_{4}$ does not depend on the external momentum and the two-loop contribution does not depend on the choice of gauge. Furthermore, Eq. (89) is transcendentally simpler than Eq. (84) as no $\zeta_{2}$ appears in Eq. 89.

\section{E. Case of massless $\mathrm{QED}_{3}$}

Finally, we consider the case of massless $\mathrm{QED}_{3}$. Such a model differs considerably from the previous ones because it is super-renormalizable. Nevertheless we shall proceed along the same lines as the previous, renormalizable, models. In $\mathrm{QED}_{3}$ we have $\varepsilon_{e}=0$ and the expansion parameter is $\delta_{\gamma}=\varepsilon_{\gamma}-1 / 2$. Eqs. (61) can then be written as:

$$
\begin{aligned}
& \Sigma_{V 1}\left(p^{2}\right)=\frac{e^{2}\left(-p^{2}\right)^{-\varepsilon_{\gamma}}}{(4 \pi)^{d_{\gamma} / 2}} \sigma_{1}\left(\varepsilon_{e}, \varepsilon_{\gamma}, a\right)=\frac{\tilde{\alpha}}{4 \pi} e^{\left(\gamma_{E}-L_{p}\right) \delta_{\gamma}} \sigma_{1}\left(\varepsilon_{e}, \varepsilon_{\gamma}, a\right), \\
& \Sigma_{V 2}\left(p^{2}\right)=\frac{e^{4}\left(-p^{2}\right)^{-2 \varepsilon_{\gamma}}}{(4 \pi)^{d_{\gamma}}} \sigma_{2}\left(\varepsilon_{e}, \varepsilon_{\gamma}, a\right)=\left(\frac{\tilde{\alpha}}{4 \pi}\right)^{2} e^{2\left(\gamma_{E}-L_{p}\right) \delta_{\gamma}} \sigma_{2}\left(\varepsilon_{e}, \varepsilon_{\gamma}, a\right) .
\end{aligned}
$$

where $L_{p}=\log \left(-p^{2} / \mu^{2}\right), \tilde{\alpha}$ is a momentum-dependent dimensionless coupling constant $\left(e^{2}\right.$ has dimension of mass in $\left.\mathrm{QED}_{3}\right)$ defined as:

$$
\tilde{\alpha}=\frac{e^{2}}{\sqrt{4 \pi} \sqrt{-p^{2}}}
$$

and we have used the fact that $z_{\alpha}=0$ for $\mathrm{QED}_{3}$, see Eq. 65). With the help of Eq. 600, the expansions read:

$$
\begin{aligned}
& e^{\left(\gamma_{E}-L_{p}\right) \delta_{\gamma}} \sigma_{1}=-\frac{a \pi^{3 / 2}}{2}+\frac{a \pi^{3 / 2}}{2}\left(L_{p}-\log (4)+2\right) \delta_{\gamma}+\mathrm{O}\left(\delta_{\gamma}^{2}\right), \\
& e^{2\left(\gamma_{E}-L_{p}\right) \delta_{\gamma}}\left(\sigma_{1}^{2}+\sigma_{2}\right)=-\frac{2 \pi N_{F}}{3 \delta_{\gamma}}+\pi\left(a^{2}+\frac{4}{9}\left(3 L_{p}-2\right) N_{F}+\frac{3 \pi^{2}}{4}-7\right)+\mathrm{O}\left(\delta_{\gamma}\right) .
\end{aligned}
$$

From these results we see that a singularity appears only at two-loop: the $1 / \delta_{\gamma}$ pole in Eq. (92b). This singularity is gauge independent and has a coefficient proportional to $N_{F}$. From Eq. 60] it can therefore be traced back to the bubble diagram which involves the master integrals $G(1,1)$ and $G\left(1, \varepsilon_{\gamma}\right)$. While $G(1,1)$ is finite in three-dimensional QED, the one-loop master integral $G\left(1, \varepsilon_{\gamma}\right)$ is indeed divergent in the limit $\varepsilon_{\gamma} \rightarrow 1 / 2$ :

$$
G\left(1, \varepsilon_{\gamma}\right)=\frac{\Gamma\left(1+\delta_{\gamma}\right)}{\sqrt{\pi}}\left[\frac{1}{\delta_{\gamma}}+6-2 \log 2+\mathrm{O}\left(\delta_{\gamma}\right)\right]
$$

At this point, the singularity looks like a UV one. Indeed, coming back to the general expression for the one-loop master integral 28) and expressing it in terms of gamma functions yields:

$$
G(\alpha, \beta)=\frac{\Gamma\left(\alpha+\beta-d_{e} / 2\right) \Gamma\left(d_{e} / 2-\alpha\right) \Gamma\left(d_{e} / 2-\beta\right)}{\Gamma(\alpha) \Gamma(\beta) \Gamma\left(d_{e}-\alpha-\beta\right)},
$$

where $d_{e}=4-2 \varepsilon_{e}-2 \varepsilon_{\gamma}$. Dimensional analysis then shows that a pole in the first gamma function in the numerator of (94) is associated with a UV singularity whereas a pole in either of the two other gamma functions in the numerator is associated with an IR singularity. For $\varepsilon_{e}=0, \alpha=1$ and $\beta=\varepsilon_{\gamma} \rightarrow 1 / 2$, we then see that the singularity is in the first gamma function of the numerator:

$$
G\left(1, \varepsilon_{\gamma}\right)=\frac{\Gamma\left(2 \varepsilon_{\gamma}-1\right) \Gamma\left(1-\varepsilon_{\gamma}\right) \Gamma\left(2-2 \varepsilon_{\gamma}\right)}{\Gamma\left(\varepsilon_{\gamma}\right) \Gamma\left(3-3 \varepsilon_{\gamma}\right)}=\frac{\Gamma\left(2 \delta_{\gamma}\right) \Gamma\left(1 / 2-\delta_{\gamma}\right) \Gamma\left(1-2 \delta_{\gamma}\right)}{\Gamma\left(1 / 2+\delta_{\gamma}\right) \Gamma\left(3 / 2-3 \delta_{\gamma}\right)}=\frac{1}{2 \delta_{\gamma}} \frac{\Gamma\left(1+2 \delta_{\gamma}\right) \Gamma\left(1 / 2-\delta_{\gamma}\right) \Gamma\left(1-2 \delta_{\gamma}\right)}{\Gamma\left(1 / 2+\delta_{\gamma}\right) \Gamma\left(3 / 2-3 \delta_{\gamma}\right)} .
$$

It turns out, however, that the present example is one in which there is an interchange between UV and IR types of singularities. Indeed, in dimensional regularization, both of these singularities correspond to poles of $\Gamma$-functions. To see the interchange, let's consider the example in more details. The above considered UV type of singularity was 
actually related to our choice of master integral: we took $G\left(1, \varepsilon_{\gamma}-\varepsilon_{e}\right)$ in $(60)$. In our calculations, however, it is the integral $G\left(1, \varepsilon_{\gamma}-\varepsilon_{e}+1\right)$ that is involved. On the one hand, it can be related to the integral $G\left(1, \varepsilon_{\gamma}-\varepsilon_{e}\right)$ by using the simple property

$$
G(1, \alpha+1)=-\frac{d_{e}-2-\alpha}{\alpha} G(1, \alpha) .
$$

On the other hand, the singularity in $G\left(1, \varepsilon_{\gamma}-\varepsilon_{e}+1\right)$ is an IR one. Indeed:

$G\left(1, \varepsilon_{\gamma}+1\right)=\frac{\Gamma\left(2 \varepsilon_{\gamma}\right) \Gamma\left(1-\varepsilon_{\gamma}\right) \Gamma\left(1-2 \varepsilon_{\gamma}\right)}{\Gamma\left(\varepsilon_{\gamma}+1\right) \Gamma\left(2-3 \varepsilon_{\gamma}\right)}=\frac{\Gamma\left(1+2 \delta_{\gamma}\right) \Gamma\left(1 / 2-\delta_{\gamma}\right) \Gamma\left(-2 \delta_{\gamma}\right)}{\Gamma\left(3 / 2+\delta_{\gamma}\right) \Gamma\left(1 / 2-3 \delta_{\gamma}\right)}=-\frac{1}{2 \delta_{\gamma}} \frac{\Gamma\left(1+2 \delta_{\gamma}\right) \Gamma\left(1 / 2-\delta_{\gamma}\right) \Gamma\left(1-2 \delta_{\gamma}\right)}{\Gamma\left(3 / 2+\delta_{\gamma}\right) \Gamma\left(1 / 2-3 \delta_{\gamma}\right)}$,

and

$$
G\left(1, \varepsilon_{\gamma}+1\right)=\frac{\Gamma\left(1+\delta_{\gamma}\right)}{\sqrt{\pi}}\left[-\frac{1}{\delta_{\gamma}}-2+2 \log 2+\mathrm{O}\left(\delta_{\gamma}\right)\right]
$$

Hence, in $\mathrm{QED}_{3}$ the singularity in the two-loop fermion self-energy is of IR origin, as it was shown earlier in Refs. [36]. This is to be contrasted with the cases of $\mathrm{QED}_{4}$ and $\mathrm{RQED}_{4,3}$ where the corresponding singularities are of the UV type.

From equations $(92)$ we find the following coefficients:

$$
\begin{aligned}
& z_{1}=0, \quad z_{20}=0, \quad z_{21}=c_{21}=-\frac{2 \pi N_{F}}{3}, \\
& r_{1}=-\frac{a \pi^{3 / 2}}{2}, \quad r_{11}=\frac{a \pi^{3 / 2}}{2}\left(L_{p}-\log (4)+2\right), \quad r_{2}=c_{22}=\pi\left(a^{2}+\frac{4}{9}\left(3 L_{p}-2\right) N_{F}+\frac{3 \pi^{2}}{4}-7\right) .
\end{aligned}
$$

This yields the following result for the renormalized fermion propagator:

$$
\begin{aligned}
-i \not p S_{r}(p ; \mu) & =1-\frac{a \sqrt{\pi}}{8} \tilde{\alpha}\left(1-\left(L_{p}-\log (4)+2\right) \delta_{\gamma}+\cdots\right)+\frac{\tilde{\alpha}^{2}}{16 \pi}\left(a^{2}+\frac{4}{9}\left(3 L_{p}-2\right) N_{F}+\frac{3 \pi^{2}}{4}-7\right)+\mathrm{O}\left(\tilde{\alpha}^{3}\right) \quad(100) \\
& =1-\frac{a e^{2}}{16 \sqrt{-p^{2}}}\left(1-\left(L_{p}-\log (4)+2\right) \delta_{\gamma}+\cdots\right)+\frac{e^{4}}{64\left(-p^{2}\right)}\left(a^{2}+\frac{4}{9}\left(3 L_{p}-2\right) N_{F}+\frac{3 \pi^{2}}{4}-7\right)+\mathrm{O}\left(e^{6}\right) .
\end{aligned}
$$

Similarly, the renormalization constant reads:

$$
Z_{\psi}=1-\frac{2 \pi N_{F}}{3 \delta_{\gamma}}\left(\frac{\tilde{\alpha}}{4 \pi}\right)^{2}+\mathrm{O}\left(\tilde{\alpha}^{3}\right)=1-\frac{N_{F}}{24 \pi \delta_{\gamma}} \tilde{\alpha}^{2}+\mathrm{O}\left(\tilde{\alpha}^{3}\right)
$$

Finally, the anomalous scaling dimension reads:

$$
\gamma_{\psi}=\frac{8 \pi N_{F}}{3}\left(\frac{\tilde{\alpha}}{4 \pi}\right)^{2}+\mathrm{O}\left(\tilde{\alpha}^{3}\right)=+\frac{N_{F}}{6 \pi} \tilde{\alpha}^{2}+\mathrm{O}\left(\tilde{\alpha}^{3}\right) .
$$

Interestingly, because there is no one-loop contribution, this anomalous dimension is fully gauge-invariant. In order to see how it affects the momentum dependence of the fermion propagator, we combine Eq. 24 with the general solution of Eq. (26). This yields:

$$
S_{r}(p ; \mu)=\frac{i}{\not p} s_{r}(1 ; \tilde{\alpha} ; a) e^{\frac{1}{2} \int_{0}^{\log \left(\frac{-p^{2}}{\mu^{2}}\right)} \mathrm{d} \log \left(\frac{-p^{\prime 2}}{\mu^{2}}\right) \gamma_{\psi}\left(\tilde{\alpha}\left(p^{\prime} ; \mu\right), a\right)},
$$

where we have taken into account the fact that, in $\mathrm{QED}_{3}, \gamma_{A}=0$ and the lack of running of the coupling constant $e^{2}$ at the considered level of accuracy. The momentum dependence of this coupling constant implies that perturbation theory is valid, i.e., $\tilde{\alpha} \ll 1$, as long as: $e^{2} \ll \sqrt{-p^{2}}$, i.e., for large euclidean momenta. With the help of Eq. 102, the asymptotic form of the dressed fermion propagator defined in the left-hand side of 103 reads:

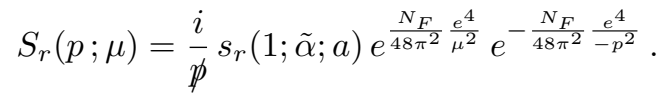

Deep in the UV, the momentum dependence of the dressed propagator is essentially the one of a free fermion in accordance with the fact that $\mathrm{QED}_{3}$ is asymptotically free. 


\section{CONCLUSION AND OUTLOOK}

The central result of this paper is the formula, Eq. 59 , for the two-loop fermion self-energy $\Sigma_{V 2}\left(p^{2}\right)$ of massless $\mathrm{RQED}_{d_{\gamma}, d_{e}}$ in an arbitrary gauge. This formula was derived using simple IBP relations. It allowed us, in the limit $d_{\gamma} \rightarrow 4$, to compute exactly $\Sigma_{V 2}\left(p^{2}\right)$ of massless $\mathrm{RQED}_{4, d_{e}}$ without calculation of the complicated two-loop diagram $G(1 / 2,1,1 / 2,1,1)$ (the last term in Eq. (59)). From Eq. (59), as well as the one-loop self-energy Eq. (34b), general expressions were derived for the fermion anomalous scaling dimension $\sqrt{79} \mathrm{p}$ and the renormalized fermion propagator, Eqs. 800 and $71 \mathrm{~b}$ ), in the limit $d_{\gamma} \rightarrow 4$. These results were then applied to $\mathrm{RQED}_{4,3}$ and compared with the cases of (renormalizable) $\mathrm{QED}_{4}$ and (super-renormalizable) $\mathrm{QED}_{3}$. In all cases, the two-loop contribution to the anomalous dimension was found to be gauge invariant. The latter was also shown to be transcendentally more complex in $\mathrm{RQED}_{4,3}$ than in usual QEDs as witnessed by the appearance of $\zeta_{2}$ in Eq. (84) with respect to Eqs. 899) and (102).

From the condensed matter physics point of view, as explained in the introduction, the massless RQED $\mathrm{R}_{4,3}$ model describes the ultra-relativistic limit of undoped graphene. Our results are a first step towards a rigorous understanding of interaction corrections to the spectral properties of graphene in the ultra-relativistic limit. Because of the Lorentz invariance of the present model there is no renormalization of the Fermi velocity ${ }^{40}$ The effect of interactions manifests at the level of the finite part of the fermion propagator as well as in the anomalous scaling dimension of the fermion field, Eq. (84). The later indicates how radiative corrections affect the momentum dependence of the dressed fermion propagator Eq. 85. For $\mathrm{RQED}_{4,3}$ our present results yield a gauge-variant dressed propagator coming from the one-loop contribution; the two-loop contribution, on the other hand, is gauge-invariant and positive (this is to be contrasted with the $\mathrm{QED}_{3}$ result which is fully gauge-invariant, see Eq. (104)). From the field theory point of view, the results obtained can be extended to an arbitrary model of massless $\mathrm{RQED}_{d_{\gamma}, d_{e}}$ with the help of an exact evaluation of $G(1 / 2,1,1 / 2,1,1)$, see App. B for general formulas. We plan to return to this task, with application to the case of $\operatorname{RQED}_{3,2}$ which requires the knowledge of the complicated contribution $G(1 / 2,1,1 / 2,1,1)$, in our future investigations.

\section{Acknowledgments}

The work of A.V.K. was supported in part by the Russian Foundation for Basic Research (Grant No. 13-02-01005) and by the Université Pierre et Marie Curie (UPMC).

\section{Appendix A: Expansion of master integrals}

Here we give the expansion of the master integrals, or $G$-functions, contributing to the fermion self-energies in Eqs. (61)-63) at fixed $\varepsilon_{e}$. The results have the following form:

$$
\begin{aligned}
& G\left(1,1-\varepsilon_{e}\right)=\frac{\exp \left[-\gamma_{E} \varepsilon_{\gamma}\right]}{\varepsilon_{\gamma}\left(1-\varepsilon_{e}-2 \varepsilon_{\gamma}\right)} \frac{1}{\Gamma\left(1-\varepsilon_{e}\right)}\left(1+\bar{\Psi}_{1} \varepsilon_{\gamma}+\frac{\varepsilon_{\gamma}^{2}}{2}\left(\bar{\Psi}_{1}^{2}+2 \zeta_{2}-3 \Psi_{2}\left(1-\varepsilon_{e}\right)\right)\right) \\
& G\left(1, \varepsilon_{\gamma}-\varepsilon_{e}\right)=-\frac{\exp \left[-\gamma_{E} \varepsilon_{\gamma}\right]}{2 \varepsilon_{\gamma}} \frac{\varepsilon_{\gamma}-\varepsilon_{e}}{\left(1-\varepsilon_{e}-3 \varepsilon_{\gamma}\right)\left(2-\varepsilon_{e}-3 \varepsilon_{\gamma}\right)} \frac{1}{\Gamma\left(1-\varepsilon_{e}\right)}\left(1+\bar{\Psi}_{1} \varepsilon_{\gamma}+\frac{\varepsilon_{\gamma}^{2}}{2}\left(\bar{\Psi}_{1}^{2}+8 \zeta_{2}-9 \Psi_{2}\left(1-\varepsilon_{e}\right)\right)\right) \\
& G\left(1-\varepsilon_{e}, \varepsilon_{\gamma}\right)=-\frac{\exp \left[-\gamma_{E} \varepsilon_{\gamma}\right]}{2\left(1-2 \varepsilon_{\gamma}\right)} \frac{1-\varepsilon_{e}-2 \varepsilon_{\gamma}}{\left(1-\varepsilon_{e}-3 \varepsilon_{\gamma}\right)\left(2-\varepsilon_{e}-3 \varepsilon_{\gamma}\right)} \frac{1}{\Gamma\left(1-\varepsilon_{e}\right)}\left(1+\bar{\Psi}_{1} \varepsilon_{\gamma}+\frac{\varepsilon_{\gamma}^{2}}{2}\left(\bar{\Psi}_{1}^{2}+4 \zeta_{2}-5 \Psi_{2}\left(1-\varepsilon_{e}\right)\right)\right) \\
& G(1,1)=\exp \left[-\gamma_{E} \varepsilon_{\gamma}\right] \frac{K_{1}}{\Gamma\left(1-\varepsilon_{e}\right)}\left(1+\bar{\Psi}_{2} \varepsilon_{\gamma}+\frac{\varepsilon_{\gamma}^{2}}{2}\left(\bar{\Psi}_{2}^{2}+\Psi_{2}\left(\varepsilon_{e}\right)+2 \Psi_{2}\left(1-\varepsilon_{e}\right)-4 \Psi_{2}\left(2-2 \varepsilon_{e}\right)\right)\right)
\end{aligned}
$$

where $\gamma_{E}$ is the Euler constant and $K_{1}, \bar{\Psi}_{1}$ and $\bar{\Psi}_{2}$ were determined in 77 .

\section{Appendix B: Exact expression of the two-loop massless propagator diagram with two non-integer indices}

In this appendix, we derive general expressions for the coefficient function $G(\alpha, 1, \beta, 1,1)$, see Eq. (39). This function can be expressed in the following form:

$$
G(\alpha, 1, \beta, 1,1)=\frac{a^{3}(1) a(\alpha) a(\beta)}{a(\alpha+\beta+3-D)} G(\tilde{\alpha}, \lambda, \tilde{\beta}, \lambda, \lambda), \quad a(\alpha)=\frac{\Gamma(\tilde{\alpha})}{\Gamma(\alpha)}, \quad \tilde{\alpha}=\frac{D}{2}-\alpha, \quad \lambda=\frac{D}{2}-1,
$$


where

$$
G(\tilde{\alpha}, \lambda, \tilde{\beta}, \lambda, \lambda)=C_{D}[\overbrace{\lambda}^{\alpha} \lambda]
$$

The function $G(\tilde{\alpha}, \lambda, \tilde{\beta}, \lambda, \lambda)$ belongs to the type of Feynman integrals considered in [13: it corresponds to the notation $C_{f}[A(\tilde{\alpha}, \tilde{\beta}, \lambda)]$ in [13] and can be considered as the particular case where $\gamma \rightarrow \lambda$ of the results in [13]. The general results contain four hypergeometric functions ${ }_{3} F_{2}$ of argument 1 . We will show that the diagram $G(\tilde{\alpha}, \lambda, \tilde{\beta}, \lambda, \lambda)$ contains only two hypergeometric functions ${ }_{3} F_{2}$ of argument 1 .

Following [13] $G(\tilde{\alpha}, \lambda, \tilde{\beta}, \lambda, \lambda)$ can be represented in the following form: $\underline{41}$

$$
G(\tilde{\alpha}, \lambda, \tilde{\beta}, \lambda, \lambda)=\frac{1}{\Gamma(\lambda)} \frac{1}{\tilde{\alpha}-1} \hat{I}(\tilde{\alpha}, \tilde{\beta}), \quad \hat{I}(\tilde{\alpha}, \tilde{\beta})=\bar{I}(\tilde{\alpha}, \tilde{\beta})-\tilde{I}(\tilde{\alpha}, \tilde{\beta}) .
$$

Taking $\gamma \rightarrow \lambda$, the results (17) in [13] becomes

$$
\begin{aligned}
& \bar{I}(\tilde{\alpha}, \tilde{\beta})=\frac{\Gamma(\lambda+1-\tilde{\beta})}{\Gamma(\tilde{\beta})}\left\{\sum _ { n = 0 } ^ { \infty } \frac { \Gamma ( n + 2 \lambda ) } { n ! \Gamma ( 2 \lambda ) } \left[\frac{1}{n+\lambda+1-\tilde{\alpha}} \frac{\Gamma(n+2-\tilde{\alpha}) \Gamma(\tilde{\alpha}+\tilde{\beta}-2)}{\Gamma(n+3+\lambda-\tilde{\alpha}-\tilde{\beta}) \Gamma(\tilde{\alpha}+\lambda-1)}\right.\right. \\
& \left.-\frac{1}{n+\lambda+\tilde{\alpha}-1}\left(\frac{\Gamma(n+1) \Gamma(\tilde{\beta}-1)}{\Gamma(n+2+\lambda-\tilde{\beta}) \Gamma(\lambda)}+\frac{\Gamma(n+\lambda-2+\tilde{\alpha}+\tilde{\beta}) \Gamma(2-\lambda-\tilde{\alpha})}{\Gamma(n-1+2 \lambda+\tilde{\alpha}) \Gamma(3-\tilde{\alpha}-\tilde{\beta})}\right)\right] \\
& \left.+\frac{\Gamma(1-\lambda) \Gamma(1+\lambda-\tilde{\alpha})}{\Gamma(2 \lambda) \Gamma(2-\tilde{\beta}) \Gamma(3-\tilde{\alpha}-\tilde{\beta})} \frac{\pi}{\sin [\pi(\lambda-1+\tilde{\beta})]}\right\},
\end{aligned}
$$

where we have used the property: $\Gamma(a) \Gamma(1-a)=\pi \sin ^{-1}[\pi a]$ for any $a$. Note that the second term in the r.h.s. of (17) in [13] has been summed in the product of $\Gamma$-functions, yielding the last term in Eq. (B4).

For the part $\tilde{I}$ there are four representations, i.e., the equations (18)-(22) in [13. We use equation (21), ${ }^{42}$ where the last terms is zero at $\gamma=\lambda$. So, we have

$$
\begin{aligned}
& \tilde{I}(\tilde{\alpha}, \tilde{\beta})=\frac{\Gamma(1-\lambda) \Gamma(1-\tilde{\beta}) \Gamma(1+\lambda-\tilde{\alpha}) \Gamma(1+\lambda-\tilde{\beta})}{\Gamma(2 \lambda) \Gamma(2-\tilde{\beta}) \Gamma(3-\tilde{\alpha}-\tilde{\beta})} \frac{\sin [\pi \tilde{\alpha}]}{\sin [\pi(\lambda-1+\tilde{\alpha})]} \\
& +\sum_{n=0}^{\infty} \frac{\Gamma(n+2 \lambda)}{n ! \Gamma(2 \lambda)} \frac{(-1)^{n}}{n+\lambda+1-\tilde{\alpha}} \frac{\Gamma(1-\tilde{\beta})}{\Gamma(\tilde{\beta}-\lambda)} \frac{\Gamma(\tilde{\alpha}+\tilde{\beta}-2) \Gamma(2-\lambda-\tilde{\alpha})}{\Gamma(n+3+\lambda-\tilde{\alpha}-\tilde{\beta}) \Gamma(\tilde{\alpha}-1-n)} .
\end{aligned}
$$

Now, we consider the difference $\bar{I}-\tilde{I}$, i.e., $\hat{I}(\tilde{\alpha}, \tilde{\beta})$. The first term and the second one in the r.h.s. of $\mathrm{B} 4$ ) and $\mathrm{B} 5$, respectively, combine to one term. The terms without series also combine to one term. So, we have

$$
\begin{aligned}
& \hat{I}(\tilde{\alpha}, \tilde{\beta})=-\sum_{n=0}^{\infty} \frac{\Gamma(n+2 \lambda)}{n ! \Gamma(2 \lambda)}\left[\frac{1}{n+\lambda+1-\tilde{\alpha}} \frac{\Gamma(n+2-\tilde{\alpha}) \Gamma(1-\tilde{\beta}) \Gamma(2-\lambda-\tilde{\alpha})}{\Gamma(n+3+\lambda-\tilde{\alpha}-\tilde{\beta}) \Gamma(3-\tilde{\alpha}-\tilde{\beta}) \Gamma(\tilde{\beta}-\lambda)} \frac{\sin [\pi \lambda]}{\sin [\pi(\lambda+1-\tilde{\beta})]}\right. \\
& \left.+\frac{\Gamma(\lambda+1-\tilde{\beta})}{\Gamma(\tilde{\beta})} \frac{1}{n+\lambda+\tilde{\alpha}-1}\left(\frac{\Gamma(n+1) \Gamma(\tilde{\beta}-1)}{\Gamma(n+2+\lambda-\tilde{\beta}) \Gamma(\lambda)}+\frac{\Gamma(n-2+\lambda+\tilde{\alpha}+\tilde{\beta}) \Gamma(2-\lambda-\tilde{\alpha})}{\Gamma(n-1+2 \lambda+\tilde{\alpha}) \Gamma(3-\tilde{\alpha}-\tilde{\beta})}\right)\right] \\
& -\frac{1}{1-\tilde{\beta}} \frac{\Gamma(1+\lambda-\tilde{\alpha}) \Gamma(1+\lambda-\tilde{\beta})}{\Gamma(2 \lambda) \Gamma(\lambda) \Gamma(3-\tilde{\alpha}-\tilde{\beta})} \frac{\pi \sin [\pi(\tilde{\beta}-\tilde{\alpha})]}{\sin [\pi(\lambda-1+\tilde{\beta})] \sin [\pi(\lambda-1+\tilde{\alpha})]}
\end{aligned}
$$

It is convenient to transform the first term in the r.h.s. to the new form containing the factor $(n+\lambda+\tilde{\alpha}-1)^{-1}$ in its denominator. It is possible to obtain from the transformation of ${ }_{3} F_{2}$ hypergeometric functions of argument 1 (see Eq. (9) in [13:

$$
\sum_{n=0}^{\infty} \frac{\Gamma(n+\hat{a}) \Gamma(n+\hat{c})}{n ! \Gamma(n+\hat{f})} \frac{1}{n+\hat{b}}=\frac{\Gamma(\hat{b}) \Gamma(\hat{c}-\hat{b})}{\Gamma(\hat{f}-\hat{b}) \Gamma(1+\hat{b}-\hat{a})} \frac{\pi}{\sin [\pi \hat{a}]}-\frac{\sin [\pi(\hat{f}-\hat{c})]}{\sin [\pi \hat{a}]} \sum_{n=0}^{\infty} \frac{\Gamma(n+\hat{c}-\hat{f}+1) \Gamma(n+\hat{c})}{n ! \Gamma(n+1+\hat{c}-\hat{a})} \frac{1}{n+\hat{c}-\hat{b}}
$$

with arbitrary $\hat{a}, \hat{b}, \hat{c}$ and $\hat{f}$. Indeed, if $\hat{c}=2 \lambda, \hat{a}=2-\tilde{\alpha}, \hat{b}=1+\lambda-\tilde{\alpha}$ and $\hat{f}=3+\lambda-\tilde{\alpha}-\tilde{\beta}$, we have

$$
\begin{aligned}
& \sum_{n=0}^{\infty} \frac{\Gamma(n+2 \lambda)}{n ! \Gamma(2 \lambda)} \frac{\Gamma(n+2-\tilde{\alpha})}{\Gamma(n+3+\lambda-\tilde{\alpha}-\tilde{\beta})} \frac{1}{n+\lambda+1-\tilde{\alpha}}=\frac{\Gamma(1+\lambda-\tilde{\alpha}) \Gamma(\lambda+\tilde{\alpha}-1)}{\Gamma(2 \lambda) \Gamma(\lambda) \Gamma(2-\tilde{\beta})} \frac{\pi}{\sin [\pi(\tilde{\alpha}-1)]} \\
& -\frac{\sin [\pi(\lambda+\tilde{\alpha}+\tilde{\beta}-2)]}{\sin [\pi(\tilde{\alpha}-1)]} \sum_{n=0}^{\infty} \frac{\Gamma(n+2 \lambda)}{n ! \Gamma(2 \lambda)} \frac{\Gamma(n-2+\lambda+\tilde{\alpha}+\tilde{\beta})}{\Gamma(n+2 \lambda+\tilde{\alpha}-1)} \frac{1}{n+\lambda+\tilde{\alpha}-1} .
\end{aligned}
$$


Taking together this new results with the last two terms in Eq. (B6), we have:

$$
\hat{I}(\tilde{\alpha}, \tilde{\beta})=\frac{\Gamma(\lambda+1-\tilde{\beta})}{\Gamma(2 \lambda) \Gamma(\lambda)} \frac{1}{(1-\tilde{\beta})} I(\tilde{\alpha}, \tilde{\beta})
$$

where

$$
\begin{aligned}
& I(\tilde{\alpha}, \tilde{\beta})=\frac{\Gamma(1+\lambda-\tilde{\alpha})}{\Gamma(3-\tilde{\alpha}-\tilde{\beta})} \frac{\pi \sin [\pi(\tilde{\beta}-\tilde{\alpha}+\lambda)]}{\sin [\pi(\lambda-1+\tilde{\beta})] \sin [\pi \tilde{\alpha}]}+\sum_{n=0}^{\infty} \frac{\Gamma(n+2 \lambda)}{n !(n+\lambda+\tilde{\alpha}-1)} \\
& \times\left(\frac{\Gamma(n+1)}{\Gamma(n+2+\lambda-\tilde{\beta})}-\frac{\Gamma(n-2+\lambda+\tilde{\alpha}+\tilde{\beta}) \Gamma(2-\tilde{\beta}) \Gamma(\lambda)}{\Gamma(n-1+2 \lambda+\tilde{\alpha}) \Gamma(3-\tilde{\alpha}-\tilde{\beta}) \Gamma(\lambda+\tilde{\alpha}-1)} \frac{\sin [\pi(\tilde{\beta}+\lambda-1)]}{\sin [\pi \tilde{\alpha}]}\right) .
\end{aligned}
$$

The results $(\mathrm{B} 9)$ and $(\mathrm{B} 10)$ together with $(\mathrm{B} 1)$ and $(\mathrm{B} 2)$ can be considered as the final result for the initial diagram $G(\alpha, 1, \beta, 1,1)$ :

$$
G(\alpha, 1, \beta, 1,1)=\frac{1}{\tilde{\alpha}-1} \frac{1}{1-\tilde{\beta}} \frac{\Gamma(\tilde{\alpha}) \Gamma(\tilde{\beta}) \Gamma(3-\tilde{\alpha}-\tilde{\beta})}{\Gamma(\alpha) \Gamma(\lambda-2+\tilde{\alpha}+\tilde{\beta})} \frac{\Gamma(\lambda)}{\Gamma(2 \lambda)} I(\tilde{\alpha}, \tilde{\beta})
$$

However, in the case where $\alpha$ and $\beta$ are close to $\lambda$, it is not so convenient because $($ B10 contains several additional singularities, which are canceled only at the end of calculations.

Another form of the final result can be obtained by application of the transformation (B7) to the last term in Eq. B10. Some simple algebra yields the following expression:

$$
\begin{aligned}
& I(\tilde{\alpha}, \tilde{\beta})=\frac{\Gamma(1+\lambda-\tilde{\alpha})}{\Gamma(3-\tilde{\alpha}-\tilde{\beta})} \frac{\pi \sin [\pi \tilde{\alpha}]}{\sin [\pi(\lambda-1+\tilde{\beta})] \sin [\pi(\tilde{\alpha}+\tilde{\beta}+\lambda-1)]}+\sum_{n=0}^{\infty} \frac{\Gamma(n+2 \lambda)}{n !}\left(\frac{1}{n+\lambda+\tilde{\alpha}-1} \frac{\Gamma(n+1)}{\Gamma(n+2+\lambda-\tilde{\beta})}\right. \\
& \left.+\frac{1}{n+\lambda+1-\tilde{\alpha}} \frac{\Gamma(n+2-\tilde{\alpha}) \Gamma(2-\tilde{\beta}) \Gamma(\lambda)}{\Gamma(n+3+\lambda-\tilde{\alpha}-\tilde{\beta}) \Gamma(3-\tilde{\alpha}-\tilde{\beta}) \Gamma(\lambda+\tilde{\alpha}-1)} \frac{\sin [\pi(\tilde{\beta}+\lambda-1)]}{\sin [\pi(\tilde{\alpha}+\tilde{\beta}+\lambda-1)]}\right) .
\end{aligned}
$$

It seems that the result (B12) together with Eq. (B11) is the most convenient final form of the result for the initial diagram $G(\alpha, 1, \beta, 1,1)$.

There are two other forms of the result for $I(\tilde{\alpha}, \tilde{\beta})$, which can be obtained by application of the transform $\mathrm{B} 7$ to the second term in Eqs. (B10) and (B12). That leads to

$$
\sum_{n=0}^{\infty} \frac{\Gamma(n+2 \lambda)}{\Gamma(n+2+\lambda-\tilde{\beta})} \frac{1}{n+\lambda+\tilde{\alpha}-1}=\frac{\sin [\pi(\tilde{\beta}+\lambda-1)]}{\pi} \sum_{n=0}^{\infty} \frac{\Gamma(n+\tilde{\beta}+\lambda-1)}{n !(n+\lambda+1-\tilde{\alpha})} \Psi_{1}(n+2 \lambda)-\frac{\Gamma(1+\lambda-\tilde{\alpha})}{\Gamma(3-\tilde{\alpha}-\tilde{\beta})} \Psi_{1}(\lambda+\tilde{\alpha}-1)
$$

Using this formula the expressions of $I(\tilde{\alpha}, \tilde{\beta})$ become more cumbersome because they contain the Euler (digamma) $\Psi_{1}$-functions. Nevertheless, we also present them for completeness. They are following

$$
\begin{aligned}
& I(\tilde{\alpha}, \tilde{\beta})=\frac{\Gamma(1+\lambda-\tilde{\alpha})}{\Gamma(3-\tilde{\alpha}-\tilde{\beta})} \frac{\pi \sin [\pi(\tilde{\beta}-\tilde{\alpha}+\lambda)]}{\sin [\pi(\lambda-1+\tilde{\beta})] \sin [\pi \tilde{\alpha}]}-\frac{\Gamma(1+\lambda-\tilde{\alpha})}{\Gamma(3-\tilde{\alpha}-\tilde{\beta})} \Psi_{1}(\lambda+\tilde{\alpha}-1) \\
& +\sum_{n=0}^{\infty} \frac{\Gamma(n+\tilde{\beta}+\lambda-1)}{n !(n+\lambda+1-\tilde{\alpha})} \Psi_{1}(n+2 \lambda) \frac{\sin [\pi(\tilde{\beta}+\lambda-1)]}{\pi} \\
& -\sum_{n=0}^{\infty} \frac{\Gamma(n+2 \lambda)}{n !(n+\lambda+\tilde{\alpha}-1)} \frac{\Gamma(n-2+\lambda+\tilde{\alpha}+\tilde{\beta}) \Gamma(2-\tilde{\beta}) \Gamma(\lambda)}{\Gamma(n-1+2 \lambda+\tilde{\alpha}) \Gamma(3-\tilde{\alpha}-\tilde{\beta}) \Gamma(\lambda+\tilde{\alpha}-1)} \frac{\sin [\pi(\tilde{\beta}+\lambda-1)]}{\sin [\pi \tilde{\alpha}]} .
\end{aligned}
$$

and

$$
\begin{aligned}
& I(\tilde{\alpha}, \tilde{\beta})=\frac{\Gamma(1+\lambda-\tilde{\alpha})}{\Gamma(3-\tilde{\alpha}-\tilde{\beta})} \frac{\pi \sin [\pi \tilde{\alpha}]}{\sin [\pi(\lambda-1+\tilde{\beta}) \sin [\pi(\tilde{\alpha}+\tilde{\beta}+\lambda-1)]}-\frac{\Gamma(1+\lambda-\tilde{\alpha})}{\Gamma(3-\tilde{\alpha}-\tilde{\beta})} \Psi_{1}(\lambda+\tilde{\alpha}-1) \\
& +\sum_{n=0}^{\infty} \frac{\Gamma(n+2 \lambda)}{n !(n+\lambda+1-\tilde{\alpha})}\left(\frac{\Gamma(n+2-\tilde{\alpha}) \Gamma(2-\tilde{\beta}) \Gamma(\lambda)}{\Gamma(n+3+\lambda-\tilde{\alpha}-\tilde{\beta}) \Gamma(3-\tilde{\alpha}-\tilde{\beta}) \Gamma(\lambda+\tilde{\alpha}-1)} \frac{\sin [\pi(\tilde{\beta}+\lambda-1)]}{\sin [\pi(\tilde{\alpha}+\tilde{\beta}+\lambda-1)]}\right. \\
& \left.+\frac{\Gamma(n+\tilde{\beta}+\lambda-1)}{\Gamma(n+2 \lambda)} \Psi_{1}(n+2 \lambda) \frac{\sin [\pi(\tilde{\beta}+\lambda-1)]}{\pi}\right) .
\end{aligned}
$$


Unfortunately, the case $\alpha=\beta$ (i.e., $\tilde{\alpha}=\tilde{\beta})$ does not produce any additional simplification and, in this case, we should use the combination of the equations $(\sqrt{\mathrm{B} 9})$ and $(\mathrm{B} 10)$ together with $\sqrt{\mathrm{B} 1})$ and $(\mathrm{B} 2)$.

1 G. E. Volovik, The Universe in a Helium Droplet, OUP Oxford (2009).

${ }^{2}$ V. N. Kotov, B. Uchoa, V. M. Pereira, F. Guinea and A. H. Castro Neto, Rev. Mod. Phys. 841067 (2012).

3 P. K. Wallace, Phys. Rev. 71622 (1947).

${ }^{4}$ G. W. Semenoff, Phys. Rev. Lett. 532449 (1984).

5 J. González, F. Guinea and M. A. H. Vozmediano, Nucl. Phys. B 424595 (1994).

${ }^{6}$ E. V. Gorbar, V. P. Gusynin and V. A. Miransky, Phys. Rev. D 64105028 (2001).

7 E. C. Marino, Nucl. Phys. B408, 551 (1993).

8 V. S. Alves, W. S. Elias, L. O. Nascimento, V. Juričić and F. Peña, Phys. Rev. D 87, 125002 (2013).

9 C. Itzykson and J.-B. Zuber, Quantum Field Theory, Dover Publications (2005);

10 M. E. Peskin and D. V. Schroeder, An Introduction to Quantum Field Theory, Perseus Books Publishing (1995).

11 A. G. Grozin, Lectures on QED and QCD, World Scientific (2007). Short version: hep-ph/0508242.

12 K. G. Chetyrkin, A. L. Kataev, F. V. Tkachov, Nucl. Phys. B174 345 (1980).

13 A. V. Kotikov, Phys. Lett. B 375240 (1996).

14 A. N. Vasil'ev, Yu. M. Pismak and Yu. R. Khonkonen, TMF 47291 (1981) [Theor. Math. Phys. 47465 (1981)].

15 K. G. Chetyrkin and F. V. Tkachov, Nucl. Phys. B192 159 (1981); F. V. Tkachov, Phys. Lett. 100B 65 (1981).

16 N. I. Usyukina, TMF 54124 (1983) [Theor. Math. Phys. 5478 (1983)].

17 D. I. Kazakov, TMF 58343 (1984) [Theor. Math. Phys. 58223 (1984)].

18 D. I. Kazakov, TMF 62127 (1985) [Theor. Math. Phys. 62 84 (1985)]; Phys. Lett. B 133406 (1983).

19 D. I. Kazakov, Report No. JINR E2-84-410, 1984.

20 S. Teber, Phys. Rev. D 86025005 (2012).

21 A. V. Kotikov and S. Teber, Phys. Rev. D 87087701 (2013).

22 E. G. Mishchenko, Europhys. Lett. 8317005 (2008); I. F. Herbut, V. Juričić and O. Vafek, Phys. Rev. Lett. 100 046403 (2008); D. E. Sheehy and J. Schmalian, Phys. Rev. B 80193411 (2009); V. Juričić, O. Vafek and I. F. Herbut, Phys. Rev. B 82235402 (2010); I. Sodemann and M. M. Fogler, Phys. Rev. B 86115408 (2012).

${ }^{23}$ R. R. Nair et al., Science 3201308 (2008); K. F. Mak et al., Phys. Rev. Lett. 101196405 (2008).

24 I. F. Herbut and V. Mastropietro, Phys. Rev. B 87205445 (2013).

25 T. W. Appelquist and R. D. Pisarski, Phys. Rev. D 23 2305 (1981); T. Appelquist and U. Heinz ibid. 242169
(1981); ibid. 252620 (1982)

26 T. W. Appelquist, M. J. Bowick, D. Karabali and L. C. R. Wijewardhana, Phys. Rev. D 333704 (1986).

27 D. Nash, Phys. Rev. Lett. 623024 (1989).

28 D. V. Shirkov, Nucl. Phys. B332 425 (1990).

29 A. G. Grozin, Int. J. Mod. Phys. A 271230018 (2012).

30 I. Bierenbaum and S. Weinzierl, Eur. Phys. J. C 3267 (2003).

31 J. A. Gracey, Phys. Lett. B 277249 (1992).

32 N. A. Kivel, A. S. Stepenenko and A. N. Vasil'ev, Nucl. Phys. B 424619 (1994); A. N. Vasiliev, S. E. Derkachov, N. A. Kivel, and A. S. Stepanenko, TMF 94179 (1993) [Theor. Math. Phys. 94127 (1993)].

33 D. J. Broadhurst, J. A. Gracey, D. Kreimer, Z. Phys. C 75559 (1997).

34 D. J. Broadhurst and A. V. Kotikov, Phys. Lett. B 441 345 (1998).

35 A. A. Vladimirov, TMF 43210 (1980) [Theor. Math. Phys. 43417 (1990)].

36 R. Jackiw and S. Templeton, Phys. Rev. D 232291 (1981); E. I. Guendelman and Z. M. Radulovic, Phys. Rev. D 27 357 (1983); Phys. Rev. D 301338 (1984).

37 In the case of $\mathrm{RQED}_{4,3}$, Eq. $(6)$ yields: $D=3-N_{\gamma}-N_{e}$. The latter exactly corresponds to the result of Ref. [26], see discussion and equations below Eq. (2.20) in this paper, for $\mathrm{QED}_{3}$ in the large- $N_{F}$ limit.

38 The proof of the Ward identity, Eq. 20, in RQED follows the same steps as for usual QEDs, see, e.g., the textbooks Refs. 9 11. The reason is that the textbook proof does not depend on the index of the gauge-field propagator which may then be arbitrary.

39 The diagram $G(1,1,1,1,1)$ was actually first computed exactly in dimensional regularization with the help of the $x$ space Gegenbauer polynomial technique in Ref. 12. Subsequently, it was computed exactly with the help of IBP in Refs. 1415.

40 The renormalization of the Fermi velocity to order $e^{4}$ has been done in the recent preprint by E. Barnes, E. H. Hwang, R. Throckmorton and S. Das Sarma, arXiv:1401.7011 [cond-mat.mes-hall].

41 Notice that, because of our definition, Eq. (35), the factor $1 /(4 \pi)^{D}$, which appears explicitly in $C_{f}[A]$ in $[13$, has already been extracted from the expression of $G$.

42 Notice that there is a slip in the first term of the r.h.s. of Eq. (21) in [13: in the denominator, $\Gamma(2+\lambda-\alpha-\beta)$ should be replaced by $\Gamma(2+\lambda-\gamma-\beta)$. 